\title{
Communication, Feedbacks and Repeated Moral Hazard with Short-lived Buyers *
}

\author{
BRUNO JULLIEN \\ Toulouse School of Economics \\ CNRS, Toulouse
}

\author{
IN-UCK PARK \\ University of Bristol \\ Sungkyunkwan University
}

April 5, 2020

\begin{abstract}
We show that experience good sellers facing myopic buyers can solve the inherent moral hazard problem by communicating their observation of quality before trade, provided that communication is part of their public track record. Such cheap-talk communication, if trusted, allows market prices to reflect the actual value created, thus providing an immediate reward for the seller's effort which complements the conventional, reputational incentives. Pre-trade communication achieves maximal efficiency when truthful and the full efficiency as the noise in the seller's observation vanishes. We fully characterize the conditions for communication to improve efficiency and the extent to which it does so.(JEL Codes: C73, D83, L14)
\end{abstract}

Keywords: cheap talk, moral hazard, reputation mechanism, trust.

\section{Introduction}

Solving moral hazard amounts to finding a way to reward the agent for exerting the socially efficient effort. In long-run market environments, forward-looking sellers may be incentivized by backloaded compensation schemes that price their goods in line with the quality they delivered in the past, exploiting ex-post monitoring of effort via delivered quality (Klein and Leffler, 1981; Shapiro, 1983). ${ }^{1}$ However, such incentive schemes are impaired and fall short of achieving efficiency when buyers are short-lived and seller's effort

*The authors thank Piotr Dworczak, Jeff Ely, Alessandro Lizzeri, George Mailath, Thomas Mariotti, Dilip Mookherjee, Volker Nocke, Alessandro Pavan, Harry Pei, Larry Samuelson, Mark Satterthwaite, and Tadashi Sekiguchi for useful discussions and comments, as well as seminar participants at Boston University, Seoul National University, Universities of Arizona, Edinburgh, Kyoto and Oxford, the Workshop on Incomplete Information: Repeated Games and Cheap Talk held at Paris School of Economics (2015), and Bristol Game Theory Workshop (2016). This work was supported by the European Research Council (ERC) under the European Union's Horizon2020 research and innovation programme (grant agreement No 670494), the Agence Nationale de la Recherche under "Investing for the Future program" (grant ANR-17-EURE-0010), ANITI funded under "Investing for the Future_PIA3" program (ANR-19PI3A-0004), and the National Research Foundation of Korea Grant funded by the Korean Government (NRF-2019S1A5A2A01047656).

${ }^{1}$ See MacLeod (2007) for a review of the related literature. 
is imperfectly monitored, no matter how patient the seller is. ${ }^{2}$ We redress this deficiency by establishing that the market pricing mechanism can restore efficiency in such situations if, in addition to the past quality supplied by a seller, her pre-trade communication on current quality is also properly reflected in pricing and in the trust level bestowed on the seller. To the best of our knowledge, this is the first result showing that cheap-talk communication can help solve moral hazard by rewarding hidden action without delay.

The importance of pre-trade communication in customer relationships is well documented in the marketing literature (Agnihotri et al., 2009; Palmatier et al., 2006). But, such soft communication by sellers on their good (e.g., online sellers describing the condition of their items and salesmen providing guidance to potential buyers) has largely been overlooked in the context of incentivizing sellers to exert the socially efficient effort.

The core insight of our paper stems from the simple observation that whatever sellers may know about the quality, provided that it is truthfully communicated, can help the market price their product closer to the actual quality. This would allow immediate compensation for the seller's effort in line with the social value created, which may complement the conventional, reputational incentives for effort. As such, truthful communication of a seller's observation of quality creates an immediate "efficiency rent" for the seller, easing the incentive constraint for her to exert the efficient effort.

However, truthful communication is at odds with the short-term incentive of claiming a better quality to get a higher price, thus it must be induced via long-term incentives. This is an extra condition, in addition to inducing efficient effort via reputational motives, that further constrains the ways in which long-term incentives may be devised if truthful communication were to be accommodated. Hence, it is unclear a priori whether seller's pre-trade communication may enhance efficiency and welfare.

We characterize precisely when and how much of such welfare improvement is possible. Broadly speaking, pre-trade communication enhances efficiency and welfare so long as the effort cost is neither too small nor too big, reaching full efficiency across the entire range of effort cost as the seller's observation of quality becomes fully accurate. Two key observations buttress such positive effects of communication. First, for the seller to preserve her efficiency rent, she must sustain buyers' trust in her communication. Second, truthful communication and efficient effort are strategic complements in the sense that the former is valuable only if the latter is intended in the future. Consequently, trustworthy behavior of the seller and the market's trust reinforce each other to uphold efficiency.

These findings lend implications on how the current feedback systems widely used in

\footnotetext{
${ }^{2}$ Note that the Folk Theorem of Fudenberg, Levine, and Maskin (1994) does not apply when buyers do not engage in a long-run relationship with a particular seller (see Fudenberg, Kreps and Levine, 1990).
} 
online platforms could be improved by suitably incorporating seller's pre-sale description of items. Online marketplaces have become a ubiquitous trading channel in less than two decades after Amazon and eBay opened in 1995, notwithstanding inherently weak trust between online traders due to their anonymity. Key innovations to tackle this trust issue have been reputation and feedback mechanisms that allow traders to leave evaluations on their counterparts for prospective future traders (Dellarocas, 2003). ${ }^{3}$ Another prominent feature of online platforms is that prospective buyers rely on the soft information provided by the seller on attributes and quality of her products. Our findings underscore the importance of properly designing communication channels and rewarding faithful communication through reputation systems. ${ }^{4}$

Specifically, we analyze the effect of pre-trade communication by a seller who repeatedly produces an experience good of random binary quality subject to moral hazard, observes a noisy signal on the produced quality and may communicate about it by cheap talk before selling the good in a market of short-lived buyers. The seller's track record of past communication and delivered quality is assumed available for potential buyers, which typically is the case (or feasible) in online markets.

We represent equilibrium value as a "self-generated" value in the sense developed by Abreu, Pearce and Stacchetti (1990) and Fudenberg and Levine (1994); then we maximize the self-generated value subject to suitable incentive compatibility conditions. We characterize fully the solution of this problem which turns out to be a tractable linear program.

As a result we can clarify how the maximum achievable efficiency level varies depending on the severity of moral hazard, the cost of efficient effort, and how noisy the seller's signal is. Without communication, the maximum achievable efficiency falls short of the full efficiency level uniformly by an amount proportional to the cost of efficient effort, regardless of seller's patience. By contrast, with communication full efficiency is achieved when the noise on the seller's signal gets small, provided that she is patient enough. Therefore, pre-trade communication enhances welfare if the seller's signal is precise enough. It is also shown that the maximum efficiency with meaningful communication is achieved when the seller communicates truthfully.

As the seller's signal gets noisier, two countervailing forces contend: on the one hand, lying is more attractive as it is more likely to go undetected; on the other hand, it is less

\footnotetext{
${ }^{3}$ Cabral and Hortasçu (2010) and Klein, Lambertz and Stahl (2016) find evidence in eBay data that feedback systems alleviate moral hazard.

${ }^{4}$ As an illustration, we note that two of the four options under eBay's rating system are related to communication: 'Item as described' and 'Communication'. Amazon has a codification of the state of used goods (from 'like new' to 'acceptable') and a dedicated section for "Customer questions \& answers."
} 
attractive because the price differential reflecting the seller's signal, which is the shortterm gain from lying, dwindles. The former effect dominates except for an intermediate range of noise levels. Thus, as the seller's signal deteriorates from being perfect, the maximum efficiency achievable with truthful communication changes non-monotonically: it declines initially since truthful communication becomes harder to induce, then improves temporarily before declining again eventually.

Turning to the cost of exerting the efficient effort, almost full efficiency is achievable without communication if the cost is very low, but not with truthful communication unless the signal is perfect. This is because imperfect monitoring of communication necessitates triggering punishment with a non-negligible probability to prevent lying, causing a non-negligible departure from the full efficiency. Hence, given any positive noise level, communication improves efficiency only if the effort cost is not too small, more precisely, when it is in an interior interval bounded away from zero.

In situations of severe moral hazard, ${ }^{5}$ it can happen that this interval vanishes when the noise level in the seller's signal is in some intermediate range but reappears both when it is lower and higher (but not too high) owing to the non-monotonic effect of noise on efficiency mentioned above. Thus, an interesting, counter-intuitive implication ensues: efficiency may be enhanced by lowering the precision of the seller's observation of quality. We also provide a tight upper bound of the noise level below which communication can be beneficial, which is not very demanding generally.

We conduct our analysis presuming that the seller communicates about the observed signal, but she could also try to communicate about the effort level exerted. However, the latter communication fails to raise the maximum seller payoff because it is redundant when the effort level is correctly anticipated. In contrast, communication on signal conveys new, interim information (not embedded in the equilibrium strategy) that can be used to compensate the hidden action on the spot and thereby, boost incentives. The underlying idea could be more general: falsifiable communication of interim information correlated with the chosen effort may enhance efficiency.

This observation points to a link with the standard insight from the relational contract literature (MacLeod and Malcomson, 1989; Baker, Gibbons and Murphy, 2002; Levin, 2003), namely, that incentives could be provided for the seller by delaying reward until after the quality is realized, through ex-post bonuses paid by a buyer voluntarily either directly or via higher future prices. Such a scheme however is not viable with short-lived buyers who would renege on any promised bonus. Our analysis suggests that pre-trade

\footnotetext{
${ }^{5}$ In the sense (to be made precise later) that the efficient effort is not easy to monitor via delivered quality, hence difficult to induce.
} 
communication may allow substituting ex-post bonuses with instant bonuses based on the information revealed by the seller.

\section{Related literature}

The current paper contributes to the literature on trust and reputation. There are broadly two modelling approaches to seller reputation as a mechanism to incentivize their productive action. The first approach, elaborated by Klein and Leffler (1981) and Shapiro (1983) among others, rests on the idea that sellers are motivated to build a good track record in order to earn the trust of future buyers and thereby, a higher future income stream. If the seller's action is perfectly monitored ex-post via delivered quality, essentially a Folk Theorem obtains and full efficiency is sustained in equilibrium. We build upon this approach for the case that the seller's action is imperfectly monitored, and characterize the maximum efficiency achievable both with and without pre-trade communication.

The other one is the adverse selection-based approach pioneered by Kreps and Wilson (1982) and Milgrom and Roberts (1982). Adapted to our context, a patient seller obtains a payoff arbitrarily close to the efficient level by appearing as a "committed" type who is believed to always take the socially efficient action (cf. Fudenberg and Levine, 1992), but in equilibrium she mixes between efficient and inefficient actions and her reputation declines gradually. Positing instead an "inept" type from which a seller desires to be distinguished, Mailath and Samuelson (2001) show, inter alia, that full efficiency is sustainable if a seller's type is subject to change at any time so that the problem of dwindling reputational motive at very high reputation levels is precluded. In contrast, we show that full efficiency is achievable even without hidden seller types, by utilizing the seller's pre-trade announcement to align her income stream more closely with the value she creates. ${ }^{6}$

More broadly, we contribute to the literature on repeated games of imperfect monitoring, shaped by such influential papers as Green and Porter (1984), Fudenberg, Levine and Maskin (1994), and Abreu, Pearce and Stacchetti (1986, 1990). Compte (1998) and Kandori and Matsushima (1998) provide folk theorems for games with communication under private monitoring and long-lived players. We focus on an environment where the power of the Folk Theorem is impaired due to myopia of short-run players, as in Fudenberg and Levine (1994), and we show that efficiency can be improved via cheap-talk communication on endogenous private information.

We build upon the insight from Sobel (1986) and Morris (2001) that truthful communication may be motivated by the desire to preserve future credibility of communication. Recently, Best and Quigley (2017) study how the concern for future may enhance credi-

\footnotetext{
${ }^{6}$ Jullien and Park (2014) show that pre-trade communication permits prices to reflect quality better in pure adverse-selection settings as well.
} 
bility of a sender who sequentially persuade short-lived receivers in the sense of Kamenica and Gentzkow (2011) but without commitment capability. Our mechanism differs critically because moral hazard is key to sustaining credibility. In our model communication doesn't steer the receiver into taking a better action for the sender-given the seller's effort choice, trade always occurs and the expected price is the same with and without communication - yet helps the seller self-discipline in providing quality. As such, without moral hazard at the production level, there is no room for communication. Thus, our contribution lies in uncovering the dynamic complementarity between two forms of moral hazard: the effort in delivering quality and the credibility in communication.

Moving to the related IO literature, Athey and Bagwell (2004) and Athey, Bagwell and Sanchirico (2004) show that ex-ante communication under adverse selection improves coordination in a collusive agreement. Awaya and Krishna (2016) show that ex-post communication improves private monitoring and helps sustain higher collusive prices. Our work differs in that we focus on interim communication under imperfect public monitoring without adverse selection. Also related is Rhodes and Wilson (2016) on false advertising as seller's announcement can be interpreted as advertising. They assume exogenous penalties for lying and exogenous quality, and focus on allocative inefficiency. Inderst and Ottaviani (2009) analyze firm's internal agency problem of incentivizing sellers to advise consumers through adequate compensation, under exogenous penalties for misselling. In comparison, we focus on improving productive efficiency via endogenous penalties for lying that arise as a result of failing trust.

The paper is organized as follows. Section 2 sets up the baseline model and characterizes the equilibrium without pre-trade communication. Section 3 contains the main analysis of the equilibrium with pre-trade communication, followed by the characterization of when such communication is welfare-enhancing in Section 4. Section 5 concludes and Appendix contains deferred proofs.

\section{Equilibrium without pre-trade communication}

We start with an infinitely repeated version of a standard moral hazard model with two effort levels and two outcomes. To disentangle the effect of pure information sharing from the standard reputation effect, we abstract from adverse selection on the agent's type.

\subsection{Baseline model}

In each period $t \in \mathbb{N}$, a long-run seller privately exerts either high effort $h$ at cost $c>0$ or low effort $\ell$ at zero cost, to produce one unit of good for sale. The quality of the produced 
item, denoted by $q_{t}$, is a random variable, independent across periods, that assumes a "good" value (i.e., $q_{t}=g$ ) with a high probability $h$ if high effort was exerted in that period but with a lower probability $\ell$ otherwise where $0<\ell<h<1$, and assumes a "bad" value (i.e., $q_{t}=b$ ) with the complementary probability. We assume that $q_{t}$ is unverifiable, albeit ex-post observable, so no warranty contract is feasible on the realized quality. Note that $h$ and $\ell$ are used to denote both the effort levels and the associated probabilities of good quality being produced.

Multiple short-run, risk neutral buyers arrive afresh at the beginning of each period and leave at the end of the period. Buyers have identical consumption values of the item, which we normalize as 1 for a good quality item and 0 for a bad quality item, that is, $g=1$ and $b=0$. Buyers do not observe the realized quality of the item prior to purchase. We assume that exerting effort is socially efficient, that is,

$$
c<h-\ell
$$

As is standard in related literature (Tadelis, 1999, Mailath and Samuelson, 2002, BarIsaac, 2003, and Board and Meyer-ter-Vehn, 2013), we assume that in each period, due to competition between buyers, the item is sold to one of the buyers at a price $p_{t}$ that is equal to its expected quality based on the information shared by the buyers. One interpretation is that the good is sold through an auction (say, second-price auction), which is common practice on trading platforms. In an online appendix, we discuss implications of alternative selling mechanisms, in particular, those of posted prices.

At the end of each period, the purchaser observes the item's true quality, $q_{t}$, and publicly reports it truthfully. ${ }^{7}$ Hence, a price and quality pair $\left(p_{t}, q_{t}\right) \in[0,1] \times\{g, b\}$ will be observed publicly after every period $t \in \mathbb{N} .^{8}$ To facilitate application of the selfgeneration idea of Abreu, Pearce and Stacchetti (1986), we allow for public randomization devices between periods; however, our results hold without such randomization devices as shown in Appendix (end of the proof of Proposition 1).

Let $H_{t}$ denote the (public) history at the beginning of period $t \in \mathbb{N}$, with $H_{1}=\emptyset$ denoting the null history. Denote by $\mathcal{H}$ the set of histories. A strategy of the seller is a mapping $e: \mathcal{H} \rightarrow[0,1]$ that specifies for each history a probability $e\left(H_{t}\right)$ with which the

\footnotetext{
${ }^{7}$ This captures consumer feedback systems prevalent in online markets and is standard in related studies, e.g., Tadelis (1999), Mailath and Samuelson (2001) and Bar-Isaac (2003).

${ }^{8}$ The seller is also informed of the effort she has exerted, but this private history has no effect on the set of equilibrium payoffs. Indeed, after some public history, the seller's effort differs depending on her effort exerted in the past. Upon reaching this history, the seller's past effort is payoff-irrelevant for the continuation game. For the agent to be willing to exert $h$ after some private history of effort and $\ell$ after another private history, she has to be indifferent between the two after the given public history. Thus, it constitutes an equilibrium that she exerts high effort with the average equilibrium probability regardless of private history for this public history.
} 
seller exerts high effort in the period following that history.

Given a seller's strategy $e$, let $p\left(H_{t} \mid e\right)=e\left(H_{t}\right)(h-\ell)+\ell$ denote the expected quality of the item produced after each history $H_{t}$. Then, the seller's normalized expected payoff from an arbitrary strategy $\tilde{e}$ when consumers expect strategy $e$ from the seller is

$$
v(\tilde{e} \mid e)=(1-\delta) \cdot \mathbf{E}\left[\sum_{t=1}^{\infty} \delta^{t-1}\left(p\left(H_{t} \mid e\right)-\tilde{e}\left(H_{t}\right) c\right) \mid \tilde{e}\right] .
$$

A strategy $e$ is a perfect public equilibrium (equilibrium, for short) of the baseline model described above if

$$
v(e \mid e) \geq v(\tilde{e} \mid e) \quad \text { for every strategy } \tilde{e} \text { of the seller. }
$$

\subsection{Characterization of equilibrium payoffs}

For an arbitrary equilibrium, the seller's payoff/value ${ }^{9}$ is

$$
v=(1-\delta)\left(p_{1}-e_{1} c\right)+\delta\left[\operatorname{Prob}\left(q=g \mid e_{1}\right) \cdot v_{g}+\operatorname{Prob}\left(q=b \mid e_{1}\right) \cdot v_{b}\right]
$$

where $e_{1}$ is the equilibrium probability with which the seller exerts $h$ in the first period, $p_{1}=e_{1}(h-\ell)+\ell$ is the equilibrium price in the first period, and $v_{q}$ is the continuation value after the realized first period quality is $q \in\{g, b\}$. As all equilibrium prices are no lower than $\ell$, every equilibrium value satisfies

$$
v \geq \underline{v}:=\ell \text {. }
$$

In fact, $\ell$ is the minimum equilibrium value obtained in the "trivial equilibrium" in which the seller exerts $\ell$ in every period regardless of history.

Suppose there is a maximum equilibrium value of the seller, denoted by $v^{*}$. Then, the continuation values $v_{q}$ in (3) may be replicated by a public randomization across extreme continuation equilibria (cf. Abreu, Pearce and Stacchetti, 1990, and Fudenberg and Levine, 1994): if the realized quality is $q \in\{g, b\}$ in the first period, the "maximum equilibrium" with the value $v^{*}$ ensues as the continuation equilibrium with probability $\rho_{q}$ while the trivial equilibrium with value $\underline{v}$ is triggered with probability $1-\rho_{q}$, where $\rho_{g}$ and $\rho_{b}$ satisfy

$$
v_{g}=\rho_{g} v^{*}+\left(1-\rho_{g}\right) \underline{v} \quad \text { and } \quad v_{b}=\rho_{b} v^{*}+\left(1-\rho_{b}\right) \underline{v} .
$$

With this representation, $v^{*}$ is the maximum self-generated value in the following sense: a value $v$ is self-generated by a tuple $\left(e_{1}, \rho_{g}, \rho_{b}\right) \in[0,1]^{3}$ if, assuming that the first period price is $p_{1}=e_{1}(h-\ell)+\ell$ and the continuation values are given by (4) with $v^{*}$ replaced

\footnotetext{
${ }^{9}$ As consumers have zero surplus, the value of the game and the seller's payoff coincide.
} 
by $v, e_{1}$ is optimal for the seller in the first period and $v$ is her value. Any self-generated value is clearly an equilibrium value (with public randomization).

Every self-generated value $v$ is at most its first period payoff by (3), because the continuation value is no higher than $v$ itself. Indeed, $\underline{v}=\ell$ is a value self-generated by a tuple with $e_{1}=0$ so that the period payoff is always $\ell$. Moreover, any $v>\underline{v}$ selfgenerated by a tuple, say $\left(e_{1}, \rho_{g}, \rho_{b}\right)$, must satisfy $e_{1}>0$ which implies that the seller obtains the value $v$ by exerting $h$ in the first period, that is,

$$
v=(1-\delta)\left(p_{1}-c\right)+\delta\left[h\left(\rho_{g} v+\left(1-\rho_{g}\right) \underline{v}\right)+(1-h)\left(\rho_{b} v+\left(1-\rho_{b}\right) \underline{v}\right)\right] .
$$

By rearranging, we express the self-generated value $v>\underline{v}$ as a function of $p_{1}, \rho_{g}$ and $\rho_{b}$ as

$$
v\left(p_{1}, \rho_{g}, \rho_{b}\right):=\frac{(1-\delta)\left(p_{1}-\ell-c\right)}{1-\delta\left(h \rho_{g}+(1-h) \rho_{b}\right)}+\underline{v} .
$$

For any $\left(p_{1}, \rho_{g}, \rho_{b}\right) \in(\ell, h] \times[0,1]^{2}$, the value $v\left(p_{1}, \rho_{g}, \rho_{b}\right)$ is self-generated if it is optimal for the seller to exert high effort (as well as low effort if $p_{1}<h$ ) in the first period:

$$
\delta(h-\ell)\left(\rho_{g}-\rho_{b}\right)\left(v\left(p_{1}, \rho_{g}, \rho_{b}\right)-\underline{v}\right) \geq(1-\delta) c \text { with equality if } p_{1}<h,
$$

that is, the future gain from exerting $h$ rather than $\ell$ exceeds the current cost $c$ (and is equal to $c$ if the seller mixes $h$ and $\ell$ ). Conversely, any $v>\underline{v}$ is a self-generated value if $v=v\left(p_{1}, \rho_{g}, \rho_{b}\right)$ for some $\left(p_{1}, \rho_{g}, \rho_{b}\right) \in(\ell, h] \times[0,1]^{2}$ and satisfies $(\mathrm{ICh})$.

Consequently, a maximum self-generated value $v^{*}$ exceeds $\underline{v}$ if and only if the following linear program has a solution, in which case $v^{*}$ is its value:

$$
\max _{\left(p_{1}, \rho_{g}, \rho_{b}\right) \in(\ell, h] \times[0,1]^{2}} v\left(p_{1}, \rho_{g}, \rho_{b}\right) \quad \text { subject to }(\mathrm{ICh}) .
$$

As $v\left(p_{1}, \rho_{g}, \rho_{b}\right)$ increases in all its arguments while the LHS (left-hand side) of the inequality (ICh) decreases in $\rho_{b}$, the solution (if it exists) is $p_{1}=h$ (hence the seller exerts high effort for sure), $\rho_{g}=1$, and the largest $\rho_{b}$ subject to (ICh). This solution and the optimized value, $v^{*}$, are calculated as:

$$
v^{*}=h-\frac{c(1-\ell)}{h-\ell}<h-c, \quad \rho_{g}^{*}=1 \quad \text { and } \quad \rho_{b}^{*}=\frac{\delta(h-\ell)^{2}-c(1-\delta \ell)}{\delta(h-\ell)^{2}-c \delta(1-\ell)} .
$$

It is straightforward to check that $0 \leq \rho_{b}^{*} \leq 1$ if and only if

$$
c<c^{*}:=\frac{(h-\ell)^{2}}{1-\ell} \quad \text { and } \quad \delta \geq \delta^{*}(c):=\frac{c}{(h-\ell)^{2}+c \ell},
$$

If (7) holds, therefore, the solution value to $\left(\mathrm{P}^{*}\right)$ is indeed the maximum self-generated value $v^{*}>\underline{v}$. If (7) fails, then the incentive compatibility (ICh) cannot hold for a legitimate tuple $\left(p_{1}, \rho_{g}, \rho_{b}\right)$ with $p_{1}>\ell$, hence $v^{*}=\underline{v}$. We summarize these findings in the next proposition where we also establish that equilibrium values constitute a convex set. 
Proposition 1 In the baseline model, the set of seller's equilibrium values is the interval $\left[\underline{v}, v^{*}\right]$ if $c<c^{*}$ and $\delta \in\left[\delta^{*}(c), 1\right)$, and is a singleton $\left\{v^{*}\right\}=\{\underline{v}\}$ otherwise.

Proof. In Appendix.

Proposition 1 establishes that the seller can be disciplined by appropriate rewards for good performances with higher continuation values, so long as she is patient enough and effort is not too costly. However, note from (6) that the maximum value $v^{*}$ falls short of the socially efficient level, $h-c$, uniformly by $\frac{c(1-h)}{h-\ell}$. Hence, efficiency can be improved only to a limited extent. The inevitable level of inefficiency, $\frac{c(1-h)}{h-\ell}$, is higher for a higher $c$ and also for a higher $\ell$ or a lower $h$. This ensues because, as the two effort levels get closer in terms of the quality distributions implied, the moral hazard problem intensifies in the sense that the effort choice becomes harder to monitor ex-post by the delivered quality.

\section{When pre-trade communication is possible}

We now modify the baseline model by assuming that in each period $t$, the seller observes an imperfect signal $s_{t} \in\{\mathfrak{g}, \mathfrak{b}\}$ regarding the realized quality of that period ( $\mathbb{g}$ for good and $\mathbb{b}$ for bad), which is incorrect with probability $\lambda<1 / 2 .{ }^{10}$ After observing $s_{t}$, the seller publicly announces a message $m_{t}$ from a finite set $M$ of cheap-talk messages. Then, the item is sold to one of the buyers at a price, $p_{t}$, that is equal to its expected quality based on the information shared by the buyers, including $m_{t}$. As before, the purchaser observes the item's true quality, $q_{t}$, and publicly reports it truthfully. The game modified with communication as such is referred to as the "communication model."

Clearly, the maximum value $v^{*}$ obtained in the previous section can be replicated in the communication model when the seller's messages are ignored as they carry no meaning (i.e., via the so-called "babbling" announcement). We delineate the extent to which meaningful communication can be sustained and improve social welfare by enhancing the market's ability to reward effort.

In the communication model, a history $H_{t}$ is defined as before except that it now includes the record of all past messages along with prices and qualities. A strategy of the seller is a pair $(e, a)$ of mappings where the effort strategy $e$ maps histories to probabilities of exerting high effort as before and the announcement strategy, $a: \mathcal{H} \times\{h, \ell\} \times\{\mathfrak{g}, \mathfrak{b}\} \rightarrow$ $\Delta(M)$, specifies a distribution over messages conditional on the effort exerted and the signal observed, as well as history. The expected payoff of the seller is defined analogously

\footnotetext{
${ }^{10}$ That is, $s_{t}=\mathfrak{g}($ resp. $\mathrm{b})$ with probability $1-\lambda$ conditional on the realized quality $q_{t}=g($ resp. $b)$.
} 
to the baseline model of no communication, accounting for the information transmitted by messages. We analyze perfect public equilibria.

Given that the seller's message will affect the future course of play in conjunction with the quality to be delivered, the optimal message to send depends on her posterior belief that the realized quality is good at that point (i.e., conditional on the signal observed and the effort exerted). We let $\pi_{s}$ denote this probability conditional on observing a signal $s \in\{\mathfrak{g}, \mathfrak{b}\}$ after exerting $h:$

$$
\pi_{\mathfrak{g}}=\frac{h(1-\lambda)}{h(1-\lambda)+(1-h) \lambda} \quad \text { and } \quad \pi_{\mathfrak{b}}=\frac{h \lambda}{h \lambda+(1-h)(1-\lambda)}<\pi_{\mathfrak{g}} .
$$

Similarly, let $\pi_{s}^{\prime}$ denote that after exerting $\ell$ :

$$
\pi_{\mathfrak{g}}^{\prime}=\frac{\ell(1-\lambda)}{\ell(1-\lambda)+(1-\ell) \lambda}<\pi_{\mathfrak{g}} \text { and } \pi_{\mathfrak{b}}^{\prime}=\frac{\ell \lambda}{\ell \lambda+(1-\ell)(1-\lambda)}<\pi_{\mathfrak{b}} .
$$

It will become clear shortly that the analysis depends on whether the seller's signal is precise enough so that a good signal $(s=g)$ indicates a higher average quality than a bad signal $(s=\mathbb{b})$ irrespective of the effort exerted, which is the case if and only if

$$
\pi_{\mathfrak{b}}<\pi_{\mathbb{g}}^{\prime} \Longleftrightarrow \lambda<\tilde{\lambda}:=\frac{\sqrt{(1-h) \ell}}{\sqrt{(1-h) \ell}+\sqrt{h(1-\ell)}} .
$$

\subsection{Faithfully self-generated values (FSGV)}

Clearly, $\underline{v}=\ell$ is the minimum equilibrium value in the modified game as well. We are interested in characterizing the maximum equilibrium value obtainable by a patient seller when communication is available, denoted by $\bar{v}$.

For any equilibrium with the maximum value $\bar{v}$, the continuation value after message $m \in M$ and realized quality $q \in\{g, b\}$ of period 1 , denoted by $v_{m q}$, can be replicated by a public randomization probability $x_{m q} \in[0,1]$ such that $^{11}$

$$
v_{m q}=x_{m q} \bar{v}+\left(1-x_{m q}\right) \underline{v} .
$$

Thus, $\bar{v}$ is "self-generated" in the sense described earlier: a seller's value $v$ is self-generated by a period strategy $\left(e_{1}, a_{1}\right)$ and randomization probabilities $\left(x_{m q}\right)_{m \in M, q \in\{g, b\}}$ if, assuming that the first period price is $p_{1}(m)=\mathbf{E}\left(q_{1} \mid e_{1}, a_{1}, m\right)$ and the continuation values are given by (9) with $v$ replacing $\bar{v}$, the strategy $\left(e_{1}, a_{1}\right)$ is optimal for the seller in the first period and $v$ is her value. By definition, any equilibrium value is self-generated if the continuation

\footnotetext{
${ }^{11}$ This has the flavor of tailoring the punishment to the crime (Mailath, Nocke and White, 2017) as the continuation payoff for low quality is affected by messages.
} 
values are no higher than itself.

As noted already, every $v \in\left[\underline{v}, v^{*}\right]$ continues to be a self-generated value with a trivial, babbling announcement (so that the price does not depend on the message). Characterizing $\bar{v}$, therefore, boils down to finding the maximum self-generated value obtainable with a non-babbling announcement strategy and comparing with $v^{*}$.

A key step in this analysis is to observe that if communication is necessary to obtain any value, then a weakly higher value can be self-generated by a specific period strategy, called the "faithful" strategy, described as follows: the seller exerts high effort (i.e., $e_{1}=1$ ) and reports the observed signal truthfully, that is, report $m=G$ upon observing a good signal $s=\mathbb{g}$ and $m=B$ upon observing a bad signal $s=\mathbb{b}$. ${ }^{12}$ We refer to a value self-generated by the faithful strategy (and some randomization probabilities) as a faithfully self-generated value (FSGV). To facilitate exposition, we state this result now and prove it later (in the proof of Proposition 3 in Appendix) because the proof utilizes characterizations of FSGV derived in due course.

Property 1. If $\delta$ is large enough, then for every self-generated value $v>v^{*}$, there is a $F S G V v_{F} \geq v$.

In light of this property, we characterize $\bar{v}$ by scrutinizing the maximum FSGV below.

For any FSGV $v_{F}$, so long as the seller follows the faithful strategy, the two messages $G$ and $B$ induce prices equal to the expected qualities given the respective signals, that is,

$$
p_{G}=\pi_{\mathfrak{g}}>h \quad \text { and } \quad p_{B}=\pi_{\mathfrak{b}}<h .
$$

Let us denote by a vector $\mathbf{x}=\left(x_{B g}, x_{G g}, x_{B b}, x_{G b}\right) \in[0,1]^{4}$ the probabilities that the seller continues with the faithful strategy (i.e., the trivial equilibrium is not triggered) after sending a message $m \in\{B, G\}$ and delivering quality $q \in\{g, b\}$. Then, the probability that her continuation value will be $v_{F}$ itself after delivering quality $q \in\{g, b\}$, denoted by $\rho_{q}$ as before, is

$$
\rho_{g}:=(1-\lambda) x_{G g}+\lambda x_{B g} \quad \text { and } \quad \rho_{b}:=(1-\lambda) x_{B b}+\lambda x_{G b} .
$$

Thus, $v_{F}$ satisfies the recursive equation:

$v_{F}=(1-\delta)\left[p_{B}+(h(1-\lambda)+(1-h) \lambda)\left(p_{G}-p_{B}\right)-c\right]+\delta\left[\left(h \rho_{g}+(1-h) \rho_{b}\right)\left(v_{F}-\underline{v}\right)+\underline{v}\right]$.

Given that the expected price in the first period is $h$ (the expected quality from high effort), this equation is rearranged to express $v_{F}$ in terms of $\mathbf{x}$, in particular $\rho_{g}$ and $\rho_{b}$, as

$$
v_{F}(\mathbf{x}):=\frac{(1-\delta)(h-\ell-c)}{1-\delta\left(h \rho_{g}+(1-h) \rho_{b}\right)}+\underline{v} .
$$

\footnotetext{
${ }^{12}$ Truthful reporting means that the sets of messages sent after signals $\mathbb{g}$ and $\mathfrak{b}$ in equilibrium are disjoint. Hence, we may restrict the message space to $\{G, B\}$ without loss of generality.
} 
This is the same form as the objective function in the program $\left(\mathrm{P}^{*}\right)$ without communication when $p_{1}=h$. Hence, communication raises the maximum self-generated value only if it can motivate the seller to exert high effort with a lower probability of triggering the trivial equilibrium.

We spell out the incentive compatibility conditions for $v_{F}(\mathbf{x})$ to be supported as an FSGV in the next section; then characterize the maximum FSGV in the subsequent section.

\subsection{Incentive compatibility conditions}

For $v_{F}(\mathbf{x})$ to be supported as a FSGV, the seller must find both exerting $h$ and truthful reporting of the signal optimal until the trivial equilibrium is triggered according to $\mathbf{x}$.

For an easy comparison of the optimality conditions with the case of no communication, we first write out the condition that the seller finds it unprofitable to deviate by exerting $\ell$ but following a truthful announcement strategy:

$$
\delta(h-\ell)\left(\rho_{g}-\rho_{b}\right)\left(v_{F}(\mathbf{x})-\underline{v}\right) \geq(1-\delta)\left[c-(h-\ell)(1-2 \lambda)\left(p_{G}-p_{B}\right)\right] \quad\left(\mathrm{ICh}_{G B}\right)
$$

where the LHS is the expected long-term benefit and the RHS is the net short-run cost of exerting $h$ rather than $\ell$. Note that exerting $h$ entails two effects: first, a good quality is more likely, enhancing the continuation values (the LHS); second, a good signal is more likely, boosting the current price (the second term of the RHS).

Condition $\left(\mathrm{ICh}_{G B}\right)$ is the counterpart of the optimality condition ( $\mathrm{ICh}$ ) for exerting $h$ with no communication. Comparing the RHS of the two conditions reveals that communication reduces the net short-run cost of exerting $h$ by $(h-\ell)(1-2 \lambda)\left(p_{G}-p_{B}\right)$. This is the expected gain in current price from exerting $h$ (as opposed to $\ell$ ), which arises because the price, now reflecting the interim information on quality communicated by the seller, is more aligned with the actual value created. This gain increases as the seller's information becomes more precise, asymptotically reaching the full contribution of high effort, $h-\ell$. As such, pre-trade communication creates a differential in the expected current revenue between exerting high and low effort. Such short-term incentives complement the conventional long-term incentives provided via continuation value differentials and thereby, relax the constraint for inducing high effort from $(\mathrm{ICh})$ to $\left(\mathrm{ICh}_{G B}\right) .{ }^{13}$

However, such a gain from communication comes at a cost in the form of extra constraints. First, truthful announcement must be induced after exerting high effort. Second,

\footnotetext{
${ }^{13}$ For such easing of constraint, it is essential that the seller's interim information on quality is from over and above her knowledge of the exerted effort. While the seller possesses information about the effort she exerted, communication of this information is redundant at any FSGV, because the effort level is correctly anticipated and fully reflected in the equilibrium price. In contrast, communication on signal conveys new information, lending additional scope for the market to provide incentives.
} 
communication opens up multiple ways for the seller to deviate.

We start with the condition for truthful announcement: a seller who follows the faithful strategy and has exerted effort $h$ (an $h$-seller, for short) must prefer to send the message $B$ (resp. $G$ ) upon observing a bad signal (resp. a good signal). This truth-telling condition for an $h$-seller after $s=\mathbb{b}$ is

$$
\begin{aligned}
& (1-\delta) p_{B}+\delta\left(\left[\pi_{\mathfrak{b}} x_{B g}+\left(1-\pi_{\mathfrak{b}}\right) x_{B b}\right]\left(v_{F}(\mathbf{x})-\underline{v}\right)+\underline{v}\right) \\
\geq & (1-\delta) p_{G}+\delta\left(\left[\pi_{\mathfrak{b}} x_{G g}+\left(1-\pi_{\mathfrak{b}}\right) x_{G b}\right]\left(v_{F}(\mathbf{x})-\underline{v}\right)+\underline{v}\right),
\end{aligned}
$$

which can be rewritten as

$$
\begin{gathered}
\delta\left(\pi_{\mathfrak{b}} \Delta_{g}+\left(1-\pi_{\mathfrak{b}}\right) \Delta_{b}\right)\left(v_{F}(\mathbf{x})-\underline{v}\right) \geq(1-\delta)\left(p_{G}-p_{B}\right) \\
\text { where } \Delta_{q}:=x_{B q}-x_{G q} \text { for } q \in\{b, g\} .
\end{gathered}
$$

An interpretation is that announcing $G$ rather than $B$ allows the seller to sell for a higher price but increases the likelihood of triggering the trivial equilibrium by $\Delta_{q}$ depending on the actual quality. When the signal is bad, this risk must be large enough for an $h$-seller not to mislead the market for a higher price, which is captured by (ICB).

When the signal is good (i.e., $s=\mathfrak{g}$ ), this risk must be small enough for an $h$-seller to opt to announce $G$ and get the high price $p_{G}$, which is the case if

$$
(1-\delta)\left(p_{G}-p_{B}\right) \geq \delta\left(\pi_{\mathbb{g}} \Delta_{g}+\left(1-\pi_{\mathfrak{g}}\right) \Delta_{b}\right)\left(v_{F}(\mathbf{x})-\underline{v}\right)
$$

Intuitively, announcing $G$ should pose a greater risk of triggering the trivial equilibrium when the quality turns out to be bad than when it turns out to be good. Indeed, the two conditions (ICB) and (ICG) imply that

$$
\Delta_{b} \geq \Delta_{g}
$$

Now, we return to the optimality condition for exerting $h$. Note that the optimal announcement of a seller who has deviated by exerting $\ell$ (an $\ell$-seller, for short) is determined by comparing continuation values from announcing $G$ and $B$, conditional on the signal. These are given by the same formulae as (ICB) and (ICG) but with $\pi_{s}$ replaced by $\pi_{s}^{\prime}$. Thus, (ICB) and (D) imply that an $\ell$-seller should truthfully announce $B$ after $s=\mathbb{b}$ because $\pi_{\mathfrak{b}}^{\prime}<\pi_{\mathfrak{b}}$; but she may or may not announce $s=\mathbb{g}$ truthfully depending on how large $\pi_{\mathfrak{g}}^{\prime}$ is. Hence the condition for $h$ to be the optimal effort requires, in addition to the earlier condition $\left(\mathrm{ICh}_{G B}\right)$ that an $\ell$-seller would not be better-off by announcing truthfully, that she would not better-off by announcing $B$ after both signals, either. That is,

$$
\left(1-\delta\left(\ell x_{B g}+(1-\ell) x_{B b}\right)\right)\left(v_{F}(\mathbf{x})-\underline{v}\right) \geq(1-\delta)\left(p_{B}-\ell\right)
$$


where the LHS is the loss of continuation value and the RHS is the current period payoff gain if the seller deviated by exerting $\ell$ and announcing $B$ after both signals.

To recap, the value $v_{F}(\mathbf{x})$ defined in (12) is a FSGV if and only if the four incentive compatibility conditions hold, namely, (ICB), (ICG), $\left(\mathrm{ICh}_{G B}\right)$ and $\left(\mathrm{ICh}_{B B}\right)$. In this case, we say that the FSGV is "supported by" the configuration $\mathbf{x}$.

Typically, not all four constraints bind at the maximum FSGV. Intuition suggests that it is more costly to induce truthful announcement of a bad signal than that of a good signal because the former requires compensating the seller for a low current price. In addition, truth-telling is more likely to be optimal after exerting $\ell$ as the signal becomes more precise because then the signal is the predominant source of information for the future. The next lemma, formalizing these intuitions, is useful in characterizing the maximum FSGV as it narrows down the set of randomization probabilities that may support it.

Lemma 1 If a maximum FSGV exists ${ }^{14}$, denoted by $\bar{v}_{F}$, then it is the solution value to the relaxed linear program:

$$
\bar{v}_{F}=\max _{\mathbf{x} \in[0,1]^{4}} v_{F}(\mathbf{x}) \quad \text { subject to }\left(I C h_{G B}\right),\left(I C h_{B B}\right),(I C B) \text { and }(D) .
$$

Moreover, $\bar{v}_{F}$ is supported by a configuration $\mathbf{x} \in[0,1]^{4}$ that binds (ICB), but leaves $\left(I_{C B}\right)$ slack if $\lambda<\tilde{\lambda}$ and leaves $\left(I C h_{G B}\right)$ slack if $\lambda>\widetilde{\lambda}$.

Proof. In Appendix.

As in standard pure adverse selection problems, a sorting condition holds for announcement that allows us to support the maximum FSGV by a configuration that binds (ICB). Then, provided that the monotonicity condition (D) holds, it is optimal to announce $m=G(B)$ if the posterior belief that quality is good exceeds (falls short of) $\pi_{\mathfrak{b}}$, the level at which the seller finds the two messages equivalent. With (ICB) binding, therefore, truthful announcement is optimal for an $h$-seller; and uniquely so for an $\ell$-seller if $\pi_{\mathfrak{g}}^{\prime}>\pi_{\mathbb{b}}$ (in which case $\left(\mathrm{ICh}_{B B}\right)$ is redundant). Likewise, announcing $B$ regardless of the observed signal is uniquely optimal for an $\ell$-seller if $\pi_{\mathscr{g}}^{\prime}<\pi_{\mathfrak{b}}$ (in which case $\left(\mathrm{ICh}_{G B}\right)$ is redundant).

One difference from standard pure adverse selection problems, however, is that achieving the maximum surplus may but need not require binding the incentive constraints for truthful announcement. This is because the reputational rent needed for inducing high effort may be already sufficient for inducing truthful announcement, as elaborated in due course.

\footnotetext{
${ }^{14}$ Existence ensues if the set of constraints is non-empty by compactness of the feasible set and continuity of the value.
} 


\subsection{Maximum FSGV and efficiency}

We now characterize when the maximum FSGV exists, what its nature is, and how it can be obtained, by exploring further the implications of Lemma 1 on equilibrium configuration.

Since the "punishment" payoff $\underline{v}$ is independent of the cost of effort $c$ while the value $v_{F}(\mathbf{x})$ decreases in $c$, the higher $c$ is the harder it is to sustain the faithful strategy. This is reflected in the conditions $(\mathrm{ICB}),\left(\mathrm{ICh}_{G B}\right)$ and $\left(\mathrm{ICh}_{B B}\right)$ becoming harder to satisfy as $c$ increases. This means that a FSGV exists for all $c$ below a threshold level. We show in Appendix that this threshold converges, as $\delta$ tends to 1 , to (recall that prices $p_{G}$ and $p_{B}$ are functions of $\lambda$ )

$$
\bar{c}(\lambda):=\left\{\begin{array}{c}
\min \left\{c^{*}+\frac{(1-2 \lambda)(h-\ell)(1-h)\left(p_{G}-p_{B}\right)}{1-\ell}, h-\ell-\frac{\lambda(1-h)\left(p_{G}-p_{B}\right)}{1-p_{B}}\right\} \\
h-\ell-\frac{\lambda(1-h)\left(p_{G}-p_{B}\right)}{1-p_{B}}-\frac{(1-h)\left(p_{B}-\ell\right)}{1-\ell} \text { if } \lambda \geq \tilde{\lambda} .
\end{array} \quad \text { if } \lambda \leq \tilde{\lambda},\right.
$$

Thus, a FSGV exists for large enough $\delta$ if and only if $c<\bar{c}(\lambda)$. The threshold $\bar{c}(\lambda)$ is continuous but not monotone in $\lambda$, with end values of

$$
\bar{c}(0)=h-\ell \text { and } \bar{c}(1 / 2)=c^{*}
$$

because $p_{G}-p_{B}=1$ at $\lambda=0$ and $p_{G}=p_{B}=h$ at $\lambda=1 / 2$.

To characterize the maximum FSGV for large enough $\delta$, it proves useful to know which other constraints bind at the solution $\mathbf{x}$ to $(\bar{P})$ that binds (ICB). Observe from (ICB) that $\bar{v}_{F}-\underline{v}$ exceeds a minimal rent $(1-\delta)\left(p_{G}-p_{B}\right)$ independently of the effort cost $c$. When $c$ is small enough, this minimal rent should be sufficient to incentivize the seller to exert high effort and consequently, the maximum FSGV should be achieved without binding the incentive compatibility conditions for inducing high effort, $\left(\mathrm{ICh}_{G B}\right)$ or $\left(\mathrm{ICh}_{B B}\right)$. We show in Appendix that this is indeed the case for large $\delta$ if $c<\widehat{c}(\lambda)$ where

$$
\widehat{c}(\lambda):=\left\{\begin{array}{c}
(h-\ell)\left(p_{G}-p_{B}\right)\left(1-2 \lambda+\frac{\lambda}{1-p_{B}}\right) \quad \text { if } \lambda \leq \widetilde{\lambda} \\
h-p_{B}-\frac{\lambda(1-h)\left(p_{G}-p_{B}\right)}{1-p_{B}} \text { if } \lambda \geq \tilde{\lambda}
\end{array}\right.
$$

which is a continuous function with end values of

$$
\widehat{c}(0)=h-\ell \text { and } \widehat{c}(1 / 2)=0 \text {. }
$$

Observe that $\widehat{c}(\lambda)<\bar{c}(\lambda)$ for $\lambda>\widetilde{\lambda}$ but not necessarily for $\lambda<\widetilde{\lambda}$. Depending on whether $\lambda$ is below or above $\tilde{\lambda}$, the details differ on how the maximum efficiency may be achieved. Yet, a similar general insight prevails that the most effective way is to reward good quality and truthful announcement of bad signal, as stated below. 
Proposition 2 A FSGV exists for large enough $\delta$ if and only if $c \in(0, \bar{c}(\lambda))$. In this case, the maximum FSGV, $\bar{v}_{F}$, is supported by a configuration $\overline{\mathbf{x}}=\left(\bar{x}_{B g}, \bar{x}_{G g}, \bar{x}_{B b}, \bar{x}_{G b}\right) \in[0,1]^{4}$ such that

(i) $\bar{x}_{B g}=\bar{x}_{G g}=1$ (i.e., $\bar{\rho}_{g}=1$ ) and moreover,

(ii) $\bar{x}_{B b}=1$ and $\bar{x}_{G b}<1$ that binds the truth-telling constraint (ICB) if $c \leq \widehat{c}(\lambda)$, in which case

$$
\bar{v}_{F}=h-c-\frac{(1-h) h \lambda(1-2 \lambda)}{(1-\lambda)(\lambda+h(1-2 \lambda))}, \quad \text { and }
$$

$\left(i i^{\prime}\right) \bar{x}_{B b}<1$ and $\bar{x}_{G b}<1$ that bind both (ICB) and the relevant constraint for inducing $h$, namely, $\left(I C h_{G B}\right)$ for $\lambda \leq \widetilde{\lambda}$ and $\left(I C h_{B B}\right)$ for $\lambda>\widetilde{\lambda}$, if $c>\widehat{c}(\lambda) .{ }^{15}$

Furthermore, $\bar{v}_{F} \rightarrow h-c$ as $\lambda \rightarrow 0$.

Proof. In Appendix.

Hence, unless the cost $c$ is too large the seller can be incentivized to exert high effort and truthfully disclose the interim information on quality prior to trade for an indefinite length of time. This is achieved via rewarding the seller by never triggering the trivial equilibrium provided that the delivered quality is good, and also provided that bad quality is disclosed when $c \leq \widehat{c}(\lambda)$. In this case, as the maximum surplus of $h-c$ accrues until the trivial equilibrium is triggered with a probability no higher than $(1-h) \lambda$ in each period, a lower bound for the maximum FSGV, $\bar{v}_{F}$, is obtained as

$$
\bar{v}_{F} \geq \frac{(1-\delta)(h-c)}{1-\delta(1-(1-h) \lambda)} \rightarrow h-c \text { as } \lambda \rightarrow 0 .
$$

Since $\widehat{c}(\lambda) \rightarrow h-\ell$ as $\lambda \rightarrow 0$, this means that full efficiency is achieved for all $c<h-\ell$ via pre-trade communication as the observation error $\lambda$ vanishes, which is also evident from (15). This is the case for all large enough $\delta$ (rather than asymptotically as $\delta \rightarrow 1$ ).

However, $\bar{v}_{F}$ is not monotone in $\lambda$ as can be seen easily from $\bar{v}_{F}$ in (15) being convex in $\lambda$ with a minimum at $\lambda=\breve{\lambda}:=\frac{\sqrt{h}}{1+2 \sqrt{h}}<1 / 3$. This reflects the dual effect of noisier signal on the truth-telling incentives: it discourages truth-telling because lying is more likely to go undetected but also encourages it by reducing the price differential to be exploited by lying. The latter dominates for low $\lambda$ while the former may take over for higher $\lambda$. Consequently, the maximum efficiency changes non-monotonically as the signal gets noisier. The non-monotonic effects of $\lambda$ on the incentives of truth-telling continue to hold for $c>\widehat{c}(\lambda)$.

It may be worth noting that the solution needs not be unique. In particular, when the signal is precise enough $(\lambda<\widetilde{\lambda})$ and the incentive compatibility for effort is binding $(c>$

\footnotetext{
${ }^{15}$ The formulae of $\bar{v}_{F}$ are lengthier and less intuitive to interpret for $\left(i i^{\prime}\right)$, hence are provided in Appendix.
} 
$\widehat{c}(\lambda)$ ), there exist other solutions where (ICB) is not binding and/or truthful announcement of bad signal is not penalized $\left(x_{B b}=1\right)$.

Having pinned down the maximum FSGV $\bar{v}_{F}$ in Proposition 2, we are now ready to characterize the set of equilibrium values in the communication model. Clearly, there are self-generated values associated with seller's strategies other than the faithful strategy. In particular, those obtained in Proposition 1 (i.e., without communication) can be replicated via babbling as mentioned earlier. As we show in Appendix, however, $\bar{v}_{F}$ is the upper bound of all self-generated values obtained with non-babbling strategies if $\delta$ is large enough (Property 1). Thus, the higher value of $\bar{v}_{F}$ and $v^{*}$ is the maximum self-generated value when pre-trade communication is possible and moreover, any value between $\underline{v}$ and this maximum constitutes an equilibrium value by the same logic used for the case without communication.

Proposition 3 In the communication model, for large enough $\delta$, the set of equilibrium values is the interval $\left[\underline{v}, \max \left\{v^{*}, \bar{v}_{F}\right\}\right]$ if $c<\bar{c}(\lambda)$ and $\left[\underline{v}, v^{*}\right]$ otherwise.

Proof. In Appendix.

Lemma 1 established that if efficiency can be improved by allowing for pre-trade communication, this is done at a maximum extent via a faithful strategy. As a consequence the maximum value is achieved with either faithful or no communication. Communication can help by relaxing the incentive compatibility condition for exerting high effort, thus stretching the extent to which high effort may be induced or rendering it possible to induce high effort (where it was not possible without communication). In the next section, we fully characterize the environments in which communication enhances welfare in either way.

We close this section with an illustration of the two thresholds $\bar{c}(\lambda)$ and $\widehat{c}(\lambda)$, in Figure 1-(a) for the case that $h-\ell$ is relatively large with $(h, \ell)=(0.75,0.25)$ and in Figure 1-(b)



(a) $\bar{c}(\lambda)$ and $\widehat{c}(\lambda)$ for large gap $h-\ell$

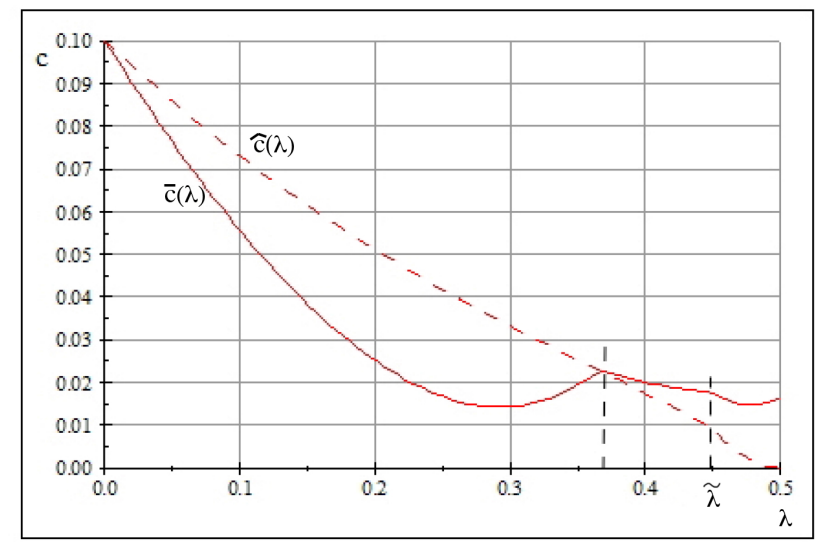

(b) $\bar{c}(\lambda)$ and $\widehat{c}(\lambda)$ for small gap $h-\ell$

Figure 1 
for the case that it is relatively small with $(h, \ell)=(0.5,0.4)$. In both diagrams, the solid curve represents $\bar{c}(\lambda)$ and the dotted curve represents $\widehat{c}(\lambda)$. Both curves are continuous

with a kink at $\lambda=\widetilde{\lambda}$. In Figure $1-(\mathrm{b}), \bar{c}(\lambda)$ has another kink below $\tilde{\lambda}$. For low $c$, a FSGV exists for all $\lambda<1 / 2$. In this case, the price differential is small when the signal is very noisy as noted above, but so is the risk differential $\Delta_{b}$ upon delivering a bad quality.

\section{When is communication beneficial?}

According to Proposition 2, pre-trade communication enhances welfare to the fully efficient level as $\lambda \rightarrow 0$ if the seller is patient enough, but impairs it as $c \rightarrow 0$ because $v^{*}$ converges to $h$ while $\bar{v}_{F}$ stays bounded away from $h$. In this section, we clarify how widespread is the welfare-enhancing effect of communication by delineating the parameter values for which a patient seller can achieve a higher value with pre-trade communication than without. We proceed by comparing $\bar{v}_{F}$ with $v^{*}$.

When $c \geq c^{*}$ so that high effort cannot be induced at all without communication, communication is clearly beneficial so long as a FSGV exists, which is the case if $c<\bar{c}(\lambda)$ by Proposition 2. We will elaborate later how large $\bar{c}(\lambda)$ is.

Consider the case that $c<c^{*}$ so that $v^{*}>\underline{v}$, meaning that high effort can be sustained without communication to some extent, in particular, with probabilities $\rho_{g}^{*}=1$ and $\rho_{b}^{*}<1$ after the seller delivered quality $q \in\{g, b\}$. In this case, communication enhances efficiency if high effort can be induced with a larger $\rho_{b}$, or equivalently, with a lower probability of triggering punishment after delivery of bad quality. This is feasible in principle because truthful communication eases the incentive constraint for inducing high effort from (ICh) to $\left(\mathrm{ICh}_{G B}\right)$ by aligning the price with the realized quality. But, inducing truthful communication adds two extra constraints $(\mathrm{ICB})$ and $\left(\mathrm{ICh}_{B B}\right)$ as explained earlier. Each constraint turns out to be a potential barrier that limits the benefits of communication in certain environments.

To derive some intuition on when the optimal configuration $\overline{\mathbf{x}}$ in Proposition 2 generates $\bar{v}_{F}$ that exceeds $v^{*}$, let us set $x_{G g}=x_{B g}=1$ (so that $\rho_{g}=1$ ) and look for the maximum $v_{F}(\mathbf{x})$ subject only to (ICB) and (D). Since the LHS of (ICB) increases in $x_{B b}$ and decreases in $x_{G b}$, this maximum value is $v_{F}(\hat{\mathbf{x}})$ where the configuration $\hat{\mathbf{x}}=\left(1,1,1, \hat{x}_{G b}\right)$ binds (ICB). For $\bar{v}_{F}>v^{*}$, therefore, it is necessary that $v_{F}(\hat{\mathbf{x}})>v^{*}$. This holds if $1-\lambda+\lambda \hat{x}_{G b}>\rho_{b}^{*}$ for all large enough $\delta$, which is verified to be the case if and only if

$$
c>\underline{c}(\lambda):=\lambda(h-\ell)\left(\frac{p_{G}-p_{B}}{1-p_{B}}\right) .
$$

To understand what goes on when $c<\underline{c}(\lambda)$, recall that inducing truthful reporting 
requires a minimal rent be given to the seller. More precisely, overstating the signal (announcing $G$ upon observing $s=\mathbb{b}$ ) is discouraged by the spread $\Delta_{b}=x_{B b}-x_{G b}$ in continuation probabilities after truthful reporting of bad quality and after overstatement. In particular, (ICB) requires that $\Delta_{b}$ be bounded away from 0 because the future loss from overstating a bad signal must overshadow the gain in current price, $p_{G}-p_{B}$. When $c$ is very small, the probability $\rho_{b}^{*}$ is very close to 1 , leaving little scope to push $x_{B b}$ above $\rho_{b}^{*}$ to have enough spread $\Delta_{b}$. Thus, truth-telling must be induced by decreasing $x_{G b}$ significantly, sacrificing efficiency. This is the barrier stemming from the truth-telling constraint (ICB).

Therefore, for communication to enhance efficiency it is necessary that $\rho_{b}^{*}$ is not too large, which is implied by (16). In fact, provided that a FSGV exists, (16) is also sufficient for communication to be beneficial if

$$
\text { either } \lambda<\tilde{\lambda} \text {, or } \lambda>\widetilde{\lambda} \text { and } c \leq \widehat{c}(\lambda) \text {. }
$$

To see this, consider the configuration $\mathbf{x}^{*}=\left(1,1,1, x_{G b}^{*}\right)$ where $1-\lambda+\lambda x_{G b}^{*}=\rho_{b}^{*}$ so that $v_{F}\left(\mathbf{x}^{*}\right)=v^{*}$. Condition (16) implies that (ICB), as well as (D), is slack at $\mathbf{x}^{*}$. Thus, $\bar{v}_{F}>v^{*}$ ensues if the incentive constraint for inducing $h$ is also slack at $\mathbf{x}^{*}$. This is indeed the case if $\lambda<\widetilde{\lambda}$ because the relevant constraint is relaxed from (ICh) to $\left(\mathrm{ICh}_{G B}\right)$. If $\lambda>\widetilde{\lambda}$ so that the relevant constraint is $\left(\mathrm{ICh}_{B B}\right)$, then $\bar{v}_{F}>v^{*}$ obtains so long as $c \leq \widehat{c}(\lambda)$ because in that case $\bar{v}_{F}$ is supported by the configuration $\hat{\mathbf{x}}$ according to Proposition 2.

In the remaining case that $\lambda>\widetilde{\lambda}$ and $c>\widehat{c}(\lambda)$, the second barrier comes into play. Specifically, at the solution configuration $\overline{\mathbf{x}}$ described in Proposition 2 where $\rho_{g}=1$, the truth-telling constraint (ICB) reduces to

$$
\Delta_{b} \geq \frac{p_{G}-p_{B}}{\left(1-p_{B}\right)(h-\ell-c)}\left(\frac{1}{\delta}-h-(1-h) \rho_{b}\right),
$$

whereas the relevant incentive constraint for inducing $h,\left(\mathrm{ICh}_{B B}\right)$, can be rewritten as (using $x_{B b}=\rho_{b}+\lambda \Delta_{b}$ )

$$
\lambda \Delta_{b} \leq\left(\frac{h-p_{B}-c}{1-\ell}\right) \frac{1-\delta}{\delta(h-\ell-c)}+\left(1-\left(\frac{p_{B}-\ell}{1-\ell}\right)\left(\frac{1-h}{h-\ell-c}\right)\right)\left(1-\rho_{b}\right) .
$$

Note that this condition prevents $\rho_{b}$ from getting too close to 1 for large $\lambda$, because $p_{B} \rightarrow h$ as $\lambda \rightarrow 1 / 2$ so that the first term on the RHS of (19) is negative. As a result, we identify in Appendix an upper bound of $\lambda$,

$$
\bar{\lambda}:=\frac{(1-h)(3 h-\ell)}{2(2 h-1)(h-\ell)}\left(\sqrt{1+\frac{4(2 h-1)(h-\ell) h}{(3 h-\ell)^{2}(1-h)}}-1\right)>\widetilde{\lambda},{ }^{16}
$$

\footnotetext{
${ }^{16}$ If $h=\frac{1}{2}$, then $\bar{\lambda}=\frac{1}{3-2 \ell}>\widetilde{\lambda}$,
} 
above which communication cannot be beneficial because $\rho_{b}>\rho_{b}^{*}$ cannot be accommodated jointly by (18) and (19). This is the barrier to beneficial communication stemming from the extra condition $\left(\mathrm{ICh}_{B B}\right)$.

It is verified straightforwardly that $\bar{c}(\lambda)$ decreases in $\lambda>\widetilde{\lambda}$ and hits $c^{*}$ at $\bar{\lambda}$. It can be further verified that $\underline{c}(\lambda)<\widehat{c}(\lambda)<\bar{c}(\lambda)$ for $\lambda \in(\widetilde{\lambda}, \bar{\lambda})$, leading to the following result on the potential for communication to improve efficiency:

Proposition $4 \bar{v}_{F}>v^{*}$ for large enough $\delta$ if and only if

$$
\lambda<\bar{\lambda} \text { and } \underline{c}(\lambda)<c<\bar{c}(\lambda)
$$

Proof. In Appendix.

The result pertains to both when $\bar{c}(\lambda) \leq c^{*}$ and when $c^{*}<\bar{c}(\lambda)$ because the latter case implies $\underline{c}(\lambda)<c^{*}$ as shown in Appendix.

According to Proposition 4 communication does not help if the signal is too unreliable $(\lambda \geq \bar{\lambda})$ or effort cost is either too small or too large. Finally, we elaborate on these boundaries at which communication ceases to be beneficial.

To get more insight about the upper bound of the observation error that allows beneficial communication, we derive the following comparative statics on $\bar{\lambda}$ :

Lemma $2 \bar{\lambda}$ increases in $\ell$, decreases in $h$, and converges to $1 / 2$ as $\ell \rightarrow h$.

Proof. In Appendix.

A higher $\ell$ and/or a lower $h$ aggravates the moral hazard problem as it degrades ex-post monitoring of effort by the delivered quality. Such changes in the environment permit less precise communication to enhance efficiency, pushing up the upper bound $\bar{\lambda}$. Note that $\bar{\lambda}$ tends to be quite large, in particular, exceeding 0.3 for $h<0.7$ even as $\ell \rightarrow 0$ where $\bar{\lambda}$ is lowest.

For a given value $\lambda<\bar{\lambda}$, the lower bound of effort cost $c$ for beneficial communication is $\underline{c}(\lambda)>0$. Note that $\underline{c}(0)=\underline{c}(0.5)=0$ and $\underline{c}(\lambda)$ is single-peaked at $\lambda=\breve{\lambda}$, the point at which $\bar{v}_{F}$ in (15) bottoms out. Such non-monotonic changes reflect the fundamental tradeoff we highlighted earlier, namely, that noisier signals reduce not only the price differential, which is the short-run gain from overstating a bad signal, but also the long-run loss by reducing the risk of getting detected.

We have shown that the range of $c$ for which communication may improve efficiency, $(\underline{c}(\lambda), \bar{c}(\lambda))$, converges to the full range $(0, h-\ell)$ as $\lambda \rightarrow 0$. Therefore, communication 
can be beneficial for the entire range of effort cost $c$ when $\lambda \rightarrow 0$; the range of $c$ gradually shrinks as $\lambda$ increases from 0 but may expand temporarily as $\lambda$ increases further before it reaches the upper bound $\bar{\lambda}$ at which the range ceases to exist.

However, if $\ell$ is sufficiently close to $h$, it may happen that $(\underline{c}(\lambda), \bar{c}(\lambda))$ is empty for some intermediate values of $\lambda \in(0, \widetilde{\lambda})$ because $\bar{c}(\lambda)$ falls below $c^{*}$ and $\underline{c}(\lambda)$ rises above $c^{*}$ at the same time. Hence, the range may disappear for some intermediate values of $\lambda<\tilde{\lambda}$ and reappear for higher values of $\lambda$. This observation leads to a counter-intuitive measure to address moral hazard in sellers: if the seller's observation of quality is not very precise and cannot be improved easily, then making it noisier may facilitate truthful communication by rendering overstatement less attractive and thereby, enhance efficiency.

In line with the discussions in this section, the grey area in Figure 3-(a) illustrates the parameter values $(c, \lambda)$ for which pre-trade communication is beneficial when $\ell$ is not too close to $h$ with the case $(h, \ell)=(0.75,0.25)$ : it is below the red curve $\bar{c}(\lambda)$ and above the black curve $\underline{c}(\lambda)$ for $\lambda$ lower than $\bar{\lambda}$ at which $\bar{c}(\lambda)$ intersects the horizontal dashed line at the level $c^{*}$. Figure 3-(b) illustrates the corresponding area when $\ell$ is closer to $h$ with $(h, \ell)=$ $(0.5,0.4)$. Notice an intermediate range of $\lambda$ where $\bar{c}(\lambda) \leq c^{*} \leq \underline{c}(\lambda)$, for which communication cannot be beneficial for any level of the effort cost $c$. Consequently, there are two disconnected regions of parameter values on which communication improves efficiency.

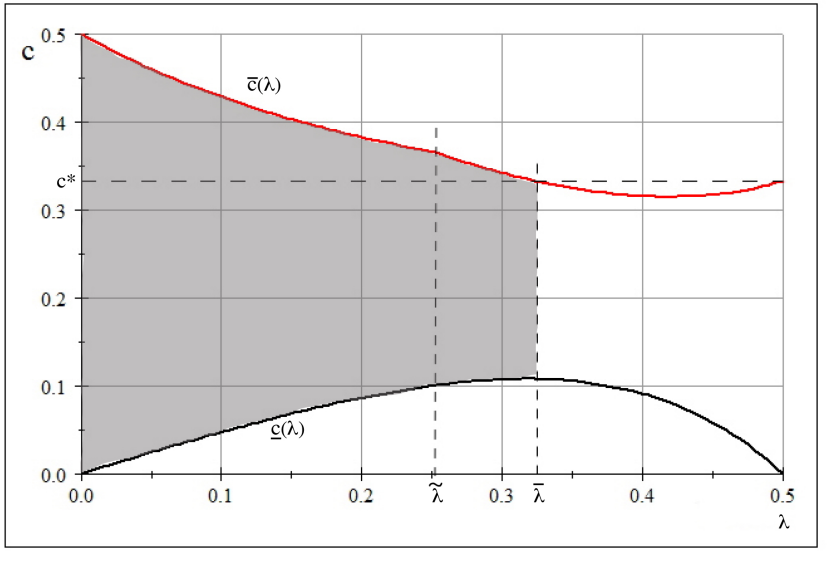

(a) Large gap $h-\ell$

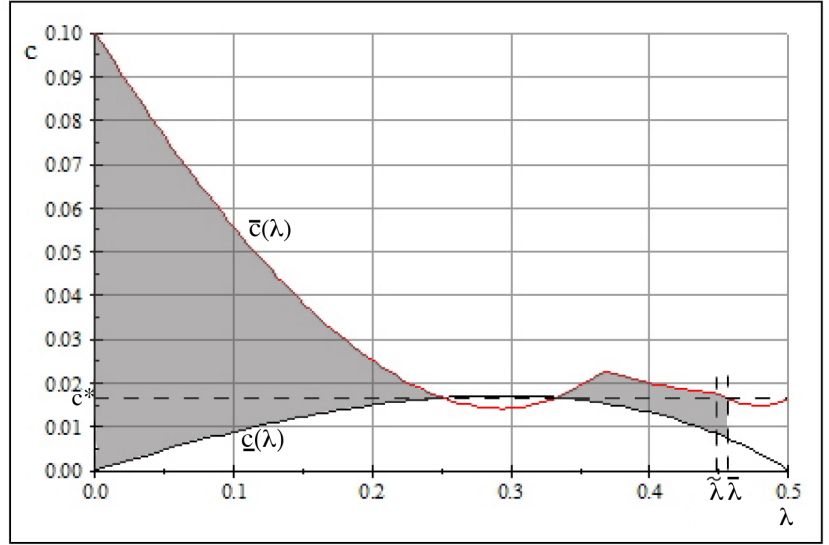

(b) Small gap $h-\ell$

Figure 3

\section{Conclusion}

In this paper we have examined how and when cheap-talk communication by a seller can help discipline herself and thereby, enhance efficiency and her equilibrium payoff. Gen- 
erally speaking, this is possible when the moral hazard problem is neither too mild nor too acute and the seller's information is not too noisy. In this case, the incentives for effort and for truthful communication are interwoven within the same reputation mechanism that determines continuation equilibria based on the seller's past performance, in such a way that truthful communication complements the conventional reputational incentives by permitting immediate reward for effort via more accurate prices. This raises the value of reputation for being trustworthy, which in turn provides credibility to seller's communication.

We have developed our analysis in a setting of experience good sellers, but the insight should be more broadly applicable to situations that involve interim communication by actors who are subject to moral hazard and reputation. For instance, managers report about likely performance, academic scholars communicate about intermediate research findings, and doctors update the progress of treatments. As already mentioned, communication could be used to move forward some reward in relational contracts.

In our model where actions, outcomes and signals are binary, the maximum equilibrium value is characterized by a well-defined linear program. This allowed us to study systematically the impacts of signal precision on the incentives for truthful communication and consequently, to characterize fully the optimal communication and effort strategy as well as the environments in which communication enhances welfare. It is an interesting agenda, which we leave for future work, to investigate whether our findings are driven by the discrete nature of our model, or they extend to richer environments that accommodate more flexible communication and actions. ${ }^{17}$

\footnotetext{
${ }^{17}$ If the seller's signal is perfect, however, our logic extends straightforwardly to the case of continua of effort/quality levels and the full efficiency is achieved.
} 


\section{Appendix}

\section{A. Proof of Proposition 1}

We have shown in the main text that the maximum self-generated value is $v^{*}$ in (6) if (7) holds, while $\underline{v}$ is the unique self-generated value if (7) fails. Thus, it remains to verify that every $v \in\left(\underline{v}, v^{*}\right)$ is an equilibrium value if $(7)$ holds.

Recall that $v^{*}$ in (6) is obtained with $p_{1}=h, \rho_{g}=1$, and $\rho_{b}=\rho_{b}^{*}$ which is the largest value compatible with (ICh). As $\left(\rho_{g}-\rho_{b}\right)\left(v\left(p_{1}, \rho_{g}, \rho_{b}\right)-\underline{v}\right)$ decreases in $\rho_{b}$, the value $v\left(h, 1, \rho_{b}\right)$ constitutes a self-generated value for all $\rho_{b} \in\left[0, \rho_{b}^{*}\right]$ as (ICh) is satisfied. Thus, any value between $v(h, 1,0)$ and $v\left(h, 1, \rho_{b}^{*}\right)$ is self-generated. Analogously, fixing $p_{1}=h$ and $\rho_{g}<1$, any value between $v\left(h, \rho_{g}, 0\right)$ and $v\left(h, \rho_{g}, \widehat{\rho}_{b}\left(\rho_{g}\right)\right)$ is self-generated, where $\widehat{\rho}_{b}\left(\rho_{g}\right)$ is the largest non-negative value compatible with (ICh) given $p_{1}=h$ and $\rho_{g}$, when it exists. Note that $\widehat{\rho}_{b}\left(\rho_{g}\right)$ increases in $\rho_{g}$ because $\left(\rho_{g}-\rho_{b}\right)\left(v\left(p_{1}, \rho_{g}, \rho_{b}\right)-\underline{v}\right)$ increases in $\rho_{g}$. Thus, there is unique $\underline{\rho_{g}}(h) \in(0,1)$ such that $\widehat{\rho}_{b}\left(\underline{\rho_{g}}(h)\right)=0$. By continuity, the set of self-generated values associated with $p_{1}=h$ is the interval $\left[v\left(h, \rho_{g}(h), 0\right), v\left(h, 1, \rho_{b}^{*}\right)\right]$.

Similarly, for each $p_{1} \in(\ell, h)$ the set of self-generated values is a closed interval ${ }^{18}$ with the minimum value of $v\left(p_{1}, \underline{\rho_{g}}\left(p_{1}\right), 0\right)$ so long as $\underline{\rho_{g}}\left(p_{1}\right) \leq 1$. As $\underline{\rho_{g}}\left(p_{1}\right)$ solves (ICh) as equality when $\rho_{b}=0$, i.e., $\underline{\rho_{g}}\left(p_{1}\right)=\frac{c}{\delta\left(c \ell+(h-\ell)\left(p_{1}-\ell\right)\right)}$, we have $\underline{\rho_{g}}\left(p_{1}\right)=\overline{1}$ when $p_{1}=\frac{c(1-\delta \ell)}{\delta(h-\ell)}+\ell$ for which $v\left(p_{1}, 1,0\right)=\widetilde{v}:=\frac{(1-\delta) c}{\delta(h-\ell)}+\underline{v}$. Therefore, the set of all self-generated values is the interval $\left[\widetilde{v}, v^{*}\right]$.

Observe from $(1-\delta) c=\delta(h-\ell)(\widetilde{v}-\underline{v})$ that the seller is indifferent between exerting $h$ and $\ell$ if the continuation payoff in period two is $\widetilde{v}$ if $q_{1}=g$ and is $\underline{v}$ if $q_{1}=b$. Given such continuation payoffs, it is optimal for the seller to exert $h$ with any probability $e_{1} \in[0,1]$, generating the seller's value of $(1-\delta) p_{1}+\delta\left(e_{1} h+\left(1-e_{1}\right) \ell\right)(\widetilde{v}-\underline{v})+\delta \underline{v}$ where $p_{1}=e_{1}(h-\ell)+\ell$. These values range from $v_{(1)}=(1-\delta) \ell+\delta(\ell(\widetilde{v}-\underline{v})+\underline{v})=\underline{v}+\delta \ell(\widetilde{v}-\underline{v})<\widetilde{v}$ to $(1-\delta) h+\delta(h(\widetilde{v}-\underline{v})+\underline{v})>\widetilde{v}$. We refer to the values in the interval $\left[v_{(1)}, \widetilde{v}\right]$ as selfgenerated with one lag. These are clearly equilibrium values.

Next, consider the seller's value from exerting $\ell$ for the first $t \geq 1$ periods, after which the continuation value is $v_{g} \in\left[v_{(1)}, \widetilde{v}\right]$ if all qualities have been good up to then but is $\underline{v}$ otherwise. As the prices are $\ell$ in the first $t$ periods, the set of seller's values obtained as such is

$$
\left.\left\{\underline{v}+\delta^{t} \ell^{t}\left(v_{g}-\underline{v}\right) \mid v_{(1)} \leq v_{g} \leq \widetilde{v}\right]\right\}=\left[\underline{v}+\delta^{t+1} \ell^{t+1}(\widetilde{v}-\underline{v}), \underline{v}+\delta^{t} \ell^{t}(\widetilde{v}-\underline{v})\right]
$$

which we call self-generated values with $t+1$ lags. For each such value $v$, the initial effort choice of $\ell$ is optimal given that the continuation value is $\underline{v}$ if $q_{1}=b$ and is a self-generated

\footnotetext{
${ }^{18}$ To be precise, it is $\left\{v\left(p_{1}, \rho_{g}, \rho_{b}\right) \mid(\mathrm{ICh})\right.$ binds for some $\left.\left(\rho_{g}, \rho_{b}\right) \in[0,1]^{2}\right\}$.
} 
value $v^{\prime}(<\tilde{v})$ with $t$ lags if $q_{1}=g$ such that $\underline{v}+\delta \ell\left(v^{\prime}-\underline{v}\right)=v$. As such, every value $v \in\left(\underline{v}, v_{(1)}\right)$ is self-generated with $t$ lags for some $t \geq 2$, which clearly is an equilibrium value.

Lastly, we show that every value $v \in\left[\underline{v}, v^{*}\right]$ is an equilibrium value even without public randomization. As shown above, any such value $v$ is either self-generated or self-generated with lags, with an associated first period strategy $e_{1}$ and a continuation value $v_{q} \in\left[\underline{v}, v^{*}\right]$ conditional on the first period quality $q \in\{g, b\}$. As each $v_{q}$ is self-generated or selfgenerated with lags, conditional on the first period quality $q \in\{g, b\}$, the associated second period strategy and subsequent continuation values are specified accordingly. Proceeding recursively, one can determine a strategy $e$ by specifying $e\left(H_{t}\right)$ for every possible history. By construction, $e\left(H_{t}\right)$ is optimal conditional on the history relative to the price schedule defined by $e$ and $v$ is the associated value, completing the proof.

\section{B. Proof of Lemma 1}

A FSGV is supported by a configuration $\mathbf{x} \in[0,1]^{4}$ that satisfies (ICB), (ICG), $\left(\mathrm{ICh}_{G B}\right)$ and $\left(\mathrm{ICh}_{B B}\right)$. Since (ICB) and (ICG) imply $(\mathrm{D})$, it follows that the solution value to $(\bar{P})$, denoted by $\overline{\bar{v}}_{F}$, is no lower than the maximum FSGV $\bar{v}_{F}$ presuming that it exists.

Consider a solution to $(\bar{P})$ which is a configuration $\mathbf{x}$ that satisfies $\left(\mathrm{ICh}_{G B}\right),\left(\mathrm{ICh}_{B B}\right)$, (ICB) and (D), so that $\Delta_{b}=x_{B b}-x_{G b}>0$. If both (ICB) and (D) are slack at $\mathbf{x}$, one can reduces $x_{B b}$ while increasing $x_{G b}$ to keep $\rho_{b}=(1-\lambda) x_{B b}+\lambda x_{G b}$ constant until either (ICB) or (D) binds. Since this keeps $v_{F}(\mathbf{x})$ and thus $\left(\mathrm{ICh}_{G B}\right)$ intact while loosening $\left(\mathrm{ICh}_{B B}\right)$, the modified configuration also supports $\overline{\bar{v}}_{F}$. Hence, we may assume that (D) or (ICB) binds at the solution $\mathbf{x}$ to $(\bar{P})$.

If only (D) binds at $\mathbf{x}$, then $x_{B q}>0$ and $x_{G q}<1$. If $x_{B g}<x_{B b}$, raise $x_{B g}$ and $x_{G g}$ by the same amount while reducing $x_{B b}$ and $x_{G b}$ by the same amount to keep $h \rho_{g}+(1-h) \rho_{b}$ intact. Since this keeps (ICB) and (D) intact and loosens $\left(\mathrm{ICh}_{G B}\right)$ and $\left(\mathrm{ICh}_{B B}\right)$, we may assume that $x_{B g} \geq x_{B b}$ at the solution $\mathbf{x}$ to $(\bar{P})$ that binds (D) but not (ICB). Then, $x_{G g}$ and $x_{G b}$ may be raised by the same amount until (ICB) binds, which clearly keeps (D) intact and loosens $\left(\mathrm{ICh}_{B B}\right)$; it also loosens $\left(\mathrm{ICh}_{G B}\right)$ because the direction of change in $\left(\rho_{g}-\rho_{b}\right)\left(v_{F}-\underline{v}\right)$ is captured by

$$
\frac{\partial}{\partial x_{G g}}\left(\left.\frac{\rho_{g}-\rho_{b}}{1-\delta\left(h \rho_{g}+(1-h) \rho_{b}\right)}\right|_{x_{G b}=x_{B b}-\Delta_{g}}\right)=\frac{1-\delta x_{B b}+\lambda\left(\delta\left(x_{B g}+x_{B b}\right)-2\right)}{\left(1-\delta\left(h \rho_{g}+(1-h) \rho_{b}\right)\right)^{2}}
$$

which is positive since it is linear in $\lambda$ and positive at both $\lambda=0$ and $1 / 2$.

Consequently, there is a solution $\mathbf{x}$ to $(\bar{P})$ that binds (ICB). Then, (D) implies that (ICG) holds at $\mathbf{x}$, further implying that $\mathbf{x}$ supports a FSGV which is at most $\bar{v}_{F}$. Since 
$\bar{v}_{F} \leq \overline{\bar{v}}_{F}$ as asserted earlier, we have established the equivalence of the maximum FSGV $\bar{v}_{F}$ and the solution value to $(\bar{P})$.

Finally, at a solution $\mathbf{x}$ to $(\bar{P})$ that binds (ICB), the seller is indifferent between announcing $G$ and $B$ when her posterior is $\pi_{\mathfrak{b}}$. Hence, upon observing $s=\mathfrak{g}$, an $\ell$ seller would find it uniquely optimal to announce $G$ (resp. $B$ ) if $\pi_{\mathfrak{b}}<\pi_{\mathbb{g}}^{\prime} \Leftrightarrow \lambda<\tilde{\lambda}$ (resp. $\pi_{\mathfrak{b}}>\pi_{\mathfrak{g}}^{\prime} \Leftrightarrow \lambda>\widetilde{\lambda}$ ) by (8). This implies that $\left(\mathrm{ICh}_{B B}\right)$ is slack at $\mathbf{x}$ if $\lambda<\tilde{\lambda}$ while $\left(\mathrm{ICh}_{G B}\right)$ is slack if $\lambda>\tilde{\lambda}$.

\section{Proof of Proposition 2}

Since $\Delta_{q}$ and $v_{F}(\mathbf{x})$ increase in $x_{B q}$ for each $q \in\{g, b\}$, so does the LHS of (ICB). Moreover, with $x_{B g}=x_{B b}=1$, the LHS of (ICB) increases in $x_{G g}$ and decreases in $x_{G b}$ for large enough $\delta$ because the respective derivative of the LHS divided by $(1-\delta)$ converges, as $\delta \rightarrow 1$, to

$\frac{h(1-h)(h-\ell-c)\left(1-x_{G b}\right)(1-2 \lambda)}{(1-\lambda-h(1-2 \lambda)) X^{2}}>0$ and $\frac{h(1-h)(h-\ell-c)\left(x_{G g}-1\right)(1-2 \lambda)}{(1-\lambda-h(1-2 \lambda)) X^{2}}<0$ where $X=\lambda\left(1-x_{G b}\right)+h\left(1-(1-\lambda) x_{G g}-\left(2-x_{G b}\right) \lambda\right)$. Hence, for (ICB) to be satisfied by some configuration, it must be satisfied by $\mathbf{x}=\left(x_{B g}, x_{G g}, x_{B b}, x_{G b}\right)=(1,1,1,0)$, which is the case if and only if

$$
c \leq h-\ell-\frac{p_{G}-p_{B}}{1-p_{B}}\left(\frac{1}{\delta}-h-(1-h)(1-\lambda)\right) \longrightarrow c_{I C B}:=h-\ell-\lambda(1-h) \frac{p_{G}-p_{B}}{1-p_{B}}
$$

as $\delta \rightarrow 1$ where the convergence is from below. Therefore,

[C1] (ICB) holds for some configuration $\mathbf{x}$ for large enough $\delta$ if and only if $c<c_{I C B}$, and in this case it holds at $\mathbf{x}=(1,1,1,0)$.

Moreover, in this case the value $v_{F}(\mathbf{x})$ is maximized subject to $(\mathrm{ICB})$ at $\hat{\mathbf{x}}=\left(1,1,1, \hat{x}_{G b}\right)$ where $\hat{x}_{G b}<1$ is the unique value at which (ICB) binds. We further establish the following.

[C2] If $\bar{v}_{F}$ is supported by a configuration $\mathbf{x}$ that leaves $\left(\mathrm{ICh}_{G B}\right)$ and $\left(\mathrm{ICh}_{B B}\right)$ slack and $x_{G b}>0$, then $\mathbf{x}=\hat{\mathbf{x}}$.

To prove this, consider a configuration $\mathbf{x}=\left(x_{B g}, x_{G g}, x_{B b}, x_{G b}\right)$ that supports $\bar{v}_{F}$ as such. We now prove [C2] in four steps.

Step 1. $x_{B g}=1$ : If $x_{B g}<1$, increase $x_{B g}$ and $x_{G g}$ slightly keeping $\Delta_{g}$ intact, which would increase $v_{F}(\mathbf{x})$ without violating any constraint of the program $(\bar{P})$. As this would contradict $\mathbf{x}$ supporting $\bar{v}_{F}$, we deduce that $x_{B g}=1$ must hold.

Step 2. $x_{G g}=1$ or $x_{B b}=1$ : If $x_{G g}<1$ and $x_{B b}<1$, one can increase $x_{G g}$ and $x_{B b}$ by the same amount, i.e., $d x_{G g}=d x_{B b}=d x>0$. If $\pi_{\mathfrak{b}}<1-\pi_{\mathfrak{b}}$, this raises $v_{F}(\mathbf{x})$ while 
relaxing $(\mathrm{ICB})$ and $(\mathrm{D})$, a contradiction to $\mathrm{x}$ supporting $\bar{v}_{F}$. If $\pi_{\mathfrak{b}}>1-\pi_{\mathfrak{b}}$, in addition to increasing $x_{G g}$ and $x_{B b}$ as above, one may reduce $x_{G b}$ so that $\left(\pi_{\mathfrak{b}} \Delta_{g}+\left(1-\pi_{\mathfrak{b}}\right) \Delta_{b}\right)$ is constant, i.e., $\left(1-2 \pi_{\mathfrak{b}}\right) d x=\left(1-\pi_{\mathfrak{b}}\right) d x_{G b}$. This increases $v_{F}(\mathbf{x})$ because

$$
d\left(h \rho_{g}+(1-h) \rho_{b}\right)=\left(1-\lambda+(1-h) \lambda \frac{1-2 \pi_{\mathfrak{b}}}{1-\pi_{\mathfrak{b}}}\right) d x>(1-2 \lambda h) d x
$$

where the inequality follows from $\frac{1-2 h}{1-h}<\frac{1-2 \pi_{\mathfrak{b}}}{1-\pi_{\mathfrak{b}}}<0$, thus relaxing (ICB) as well as (D), again a contradiction.

Step 3. $x_{G g}=x_{B b}=1$ : If $x_{G g}=1>x_{B b}$, increase $x_{B b}$. If $x_{G g}<1=x_{B b}$, increase $x_{G g}$ and decrease $x_{G b}$ in such a way that $\pi_{\mathfrak{b}} \Delta_{g}+\left(1-\pi_{\mathfrak{b}}\right) \Delta_{b}$ is intact, i.e., $\pi_{\mathfrak{b}} d x_{G g}+\left(1-\pi_{\mathfrak{b}}\right) d x_{G b}=$ 0 and thus

$$
d\left(h \rho_{g}+(1-h) \rho_{b}\right)=h(1-\lambda) d x_{G g}+(1-h) \lambda d x_{G b}>(1-\lambda)\left[h d x_{G g}+(1-h) d x_{G b}\right]>0 .
$$

Either case, $v_{F}(\mathbf{x})$ increases while maintaining (ICB) and (D), a contradiction to $\mathbf{x}$ supporting $\bar{v}_{F}$.

Step 4. $\hat{\mathbf{x}}$ supports $\bar{v}_{F}$ : By Steps $1-3, \bar{v}_{F}$ is supported by a configuration $\mathbf{x}=\left(1,1,1, x_{G b}\right)$ at which $\left(\mathrm{ICh}_{G B}\right)$ and $\left(\mathrm{ICh}_{B B}\right)$ are slack, as well as (D). Therefore, (ICB) must bind because otherwise $x_{G b}$ may be increased without violating any constraint. This proves [C2].

When [C2] applies, the solution is $\hat{\mathbf{x}}$ where

$$
\hat{x}_{G b}=\frac{\delta\left(1-p_{B}\right)(h-\ell-c)-\left(p_{G}-p_{B}\right)(1-\delta+\delta(1-h) \lambda)}{\delta\left[\left(1-p_{B}\right)(h-\ell-c)-\left(p_{G}-p_{B}\right)(1-h) \lambda\right]}
$$

and $\bar{v}_{F}(\hat{\mathbf{x}})$ is routinely calculated as the formula in (15).

The proof now proceeds differently between the two cases $\lambda \leq \widetilde{\lambda}$ and $\lambda>\widetilde{\lambda}$.

\section{C.1 Case where $\lambda \leq \widetilde{\lambda}$}

In this case we focus on $\left(\mathrm{ICh}_{G B}\right),(\mathrm{ICB})$ and $(\mathrm{D})$ because the three conditions, with (ICB) binding, imply $\left(\mathrm{ICh}_{B B}\right)$. As $v_{F}(\mathbf{x})$ increases in $\rho_{g}$, for $\left(\mathrm{ICh}_{G B}\right)$ to hold at any $\mathbf{x}$ it must holds at $\rho_{g}=1$ which is written as

$$
\frac{\delta\left(1-\rho_{b}\right)(1-h)}{\left(1-\delta h-\delta(1-h) \rho_{b}\right)} \geq \frac{\left(c-(h-\ell)(1-2 \lambda)\left(p_{G}-p_{B}\right)\right)(1-h)}{(h-\ell)(h-\ell-c)} .
$$

The LHS decreases in $\rho_{b}$, and it is less than 1 and converges to 1 as $\delta \rightarrow 1$ for all $\rho_{b}<1$. Hence, $\left(\mathrm{ICh}_{G B}\right)$ may hold at some $\mathbf{x}$ for large enough $\delta$ if and only if the RHS is strictly less than 1 , which is calculated to be the case if and only if

$$
c<c_{I C h}:=c^{*}+(1-2 \lambda) \frac{(h-\ell)(1-h)}{1-\ell}\left(p_{G}-p_{B}\right) .
$$


Note that, in this case, $\left(\operatorname{ICh}_{G B}\right)$ holds at every $\mathbf{x}=\left(1,1, x_{B b}, x_{G b}\right) \neq(1,1,1,1)$ for $\delta$ large enough because the LHS of (21) converges to 1 as $\delta \rightarrow 1$. Together with [C1], therefore, both $\left(\mathrm{ICh}_{G B}\right)$ and (ICB) hold at some $\mathbf{x}$ when $\delta$ is large enough if and only if $c<\min \left\{c_{I C h}, c_{I C B}\right\}=\bar{c}(\lambda)$. Since in this case they both hold at $\mathbf{x}=(1,1,1,0)$ which also satisfies (D), a FSGV exists for large enough $\delta$ if and only if $c \in(0, \bar{c}(\lambda))$, thus so does the maximum FSGV, $\bar{v}_{F}$, by the Maximum theorem (as the objective function of $(\bar{P})$ is continuous subject to a compact constraint set).

Suppose $c<\bar{c}(\lambda)$, so that $\bar{v}_{F}$ exists. Since $\mathbf{x}=(1,1,1,0)$ satisfies $\left(\mathrm{ICh}_{G B}\right)$, (ICB) and (D) strictly in this case, we have $\bar{v}_{F}>v_{F}(1,1,1,0)$. Thus, any configuration $\mathbf{x}=$ $\left(x_{B g}, x_{G g}, x_{B b}, x_{G b}\right)$ supporting $\bar{v}_{F}$ must have $x_{G b}>0$.

Hence, if $\bar{v}_{F}$ is supported by a configuration at which $\left(\mathrm{ICh}_{G B}\right)$ is slack, it must be $\hat{\mathbf{x}}=$ $\left(1,1,1, \hat{x}_{G b}\right)$ by $[\mathrm{C} 2]$, and thus, $\left(\mathrm{ICh}_{G B}\right)$ must be slack at $\hat{\mathbf{x}}$. Given $x_{G g}=x_{B g}=x_{B b}=1$, it is routinely verified that the LHS of $\left(\mathrm{ICh}_{G B}\right)$ decreases in $x_{G b}$, hence it binds at a unique $x_{G b}^{\prime}<1$ and hold at all lower $x_{G b}$. It is straightforward to verify (by Mathematica) that $\hat{x}_{G b} \leq x_{G b}^{\prime}$ for large enough $\delta$ if and only if

$$
c \leq(h-\ell)\left(p_{G}-p_{B}\right)\left(1-2 \lambda+\frac{\lambda}{1-p_{B}}\right)=\widehat{c}(\lambda) .
$$

This verifies that $\bar{v}_{F}$ is supported by $\mathbf{x}=\left(1,1,1, \hat{x}_{G b}\right)$ for large enough $\delta$ if $c \leq \widehat{c}(\lambda)$, establishing Proposition 2 for $\lambda \leq \widetilde{\lambda}$ when $c \leq \widehat{c}(\lambda)$.

If $c>\widehat{c}(\lambda)$, on the other hand, $\bar{v}_{F}$ cannot be supported by a configuration at which $\left(\mathrm{ICh}_{G B}\right)$ is slack by [C2], thus it is supported by $\mathbf{x}=\left(x_{B g}, x_{G g}, x_{B b}, x_{G b}\right)$ that binds $\left(\mathrm{ICh}_{G B}\right)$. Note that $\mathbf{x}^{\prime}=\left(1,1,1, x_{G b}^{\prime}\right)$ binds $\left(\mathrm{ICh}_{G B}\right)$ and satisfies (ICB) and (D) loosely. If $v_{F}(\mathbf{x})>v_{F}\left(\mathbf{x}^{\prime}\right)$, then $\rho_{b}$ must be higher at $\mathbf{x}$ than $\mathbf{x}^{\prime}$, but then the LHS of $\left(\mathrm{ICh}_{G B}\right)$ is lower at $\mathbf{x}$ than $\mathbf{x}^{\prime}$ because the LHS of $\left(\mathrm{ICh}_{G B}\right)$ increases in $\rho_{g}$ and decreases in $\rho_{b}$, a contradiction to $\mathbf{x}$ supporting $\bar{v}_{F}$. Therefore, $\bar{v}_{F}$ is supported by $\mathbf{x}^{\prime}$, and $\bar{v}_{F}\left(\mathbf{x}^{\prime}\right)$ is calculated (by Mathematica) as

$$
\bar{v}_{F}\left(\mathbf{x}^{\prime}\right)=-\frac{c(1-\ell)}{h-\ell}+\frac{1-h(1-2 \lambda)^{2}-3 \lambda(1-\lambda)}{h(1-2 \lambda)^{2}(1-h)+\lambda(1-\lambda)} h .
$$

However, since (ICB) is slack at $\mathbf{x}^{\prime}$, there is flexibility in choosing $x_{B b}$ and $x_{G b}$ because only $\rho_{b}$ matters for $v_{F}(\mathbf{x})$ and $\left(\mathrm{ICh}_{G B}\right)$. Thus we can also obtain $\bar{v}_{F}$ with (ICB) binding, by reducing $x_{B b}$ from 1 and increasing $x_{G b}$ from $x_{G b}^{\prime}$ keeping $\rho_{b}$ intact until (ICB) binds. Note that (D) holds because $\Delta_{g}=0$.

This establishes Proposition 2 for $\lambda \leq \tilde{\lambda}$.

\section{Discussion of the thresholds}

In fact, the thresholds are related as follows: 


$$
c_{I C h}-\widehat{c}(\lambda)=(h-\ell) A(\lambda) \text { and } c_{I C B}-\widehat{c}(\lambda)=(1-\ell) A(\lambda)
$$

where

$$
\begin{aligned}
A(\lambda) & :=(1-\ell)\left[\frac{h-\ell}{1-\ell}\left(1-\left(p_{G}-p_{B}\right)(1-2 \lambda)\right)-\frac{\lambda\left(p_{G}-p_{B}\right)}{1-p_{B}}\right] \\
& =\frac{\lambda h\left[\frac{h-\ell}{h(1-\ell)}(1-\lambda)^{2}-(1-2 \lambda)(h \lambda+(1-h)(1-\lambda))\right]}{(1-\lambda)(h(1-\lambda)+(1-h) \lambda)(h \lambda+(1-h)(1-\lambda)} .
\end{aligned}
$$

Hence either $\widehat{c}(\lambda)<\bar{c}(\lambda)=c_{I C h}<c_{I C B}$ or $\bar{c}(\lambda)=c_{I C B}<c_{I C h}<\widehat{c}(\lambda)$. The latter case occurs when $\left(\mathrm{ICh}_{G B}\right)$ is easier to satisfy than (ICB) so that the truth-telling rent, the RHS of (ICB), is sufficient to induce $h$. In the former case $\left(\mathrm{ICh}_{G B}\right)$ becomes more stringent than (ICB) when the effort cost $c$ is large enough.

Observe that $\widehat{c}(\lambda)<\bar{c}(\lambda)$ when $A(\lambda)>0$ and $\widehat{c}(\lambda)>\bar{c}(\lambda)$ when $A(\lambda)<0$. Note that

$r:=\frac{h-\ell}{h(1-\ell)}$ decreases from 1 to 0 as $\ell$ increases from 0 to $h$. Given any $\lambda$, in particular, $\bar{c}(\lambda)<\widehat{c}(\lambda)$ if $\ell$ is close enough to $h$.

To check the sign of $A(\lambda)$ by that of the term in the bracket of (22), we note that

$$
B(\lambda)=r \frac{(1-\lambda)^{2}}{(1-2 \lambda)}-(h \lambda+(1-h)(1-\lambda))
$$

is convex and $B(0)=r+h-1$ and $B^{\prime}(0)<0$. Since $\widetilde{\lambda}=\frac{r-1+\sqrt{1-r}}{r}$ from $\frac{h(1-r)}{1-r h}=\ell$, we get

$$
\begin{aligned}
B(\widetilde{\lambda}) & =\frac{r(1-\sqrt{1-r})^{2}}{(2-r-2 \sqrt{1-r})}-(h(r-1+\sqrt{1-r})+(1-h)(1-\sqrt{1-r})) \\
& \geq\left(\frac{r(1-\sqrt{1-r})}{2-r-2 \sqrt{1-r}}-1\right)(1-\sqrt{1-r})>0
\end{aligned}
$$

Therefore,

- If $B(0) \leq 0 \Leftrightarrow r+h \leq 1$, then we have $\widehat{c}(\lambda)<\bar{c}(\lambda)=c_{I C h}$ for $\lambda$ above some threshold while $\bar{c}(\lambda)=c_{I C B}<\widehat{c}(\lambda)$ below the threshold.

- If $r+h>1$ and $r$ is not too large, then $\widehat{c}(\lambda)<\bar{c}(\lambda)=c_{I C h}$ for $\lambda$ small or close to $\widetilde{\lambda}$, but $\bar{c}(\lambda)=c_{I C B}<\widehat{c}(\lambda)$ holds in some interior interval.

- Finally, when $r+h>1$ and $r$ is large $\left(\ell\right.$ is small) so that $\frac{(1-\lambda)^{2}}{(1-2 \lambda)}>1$, then $\widehat{c}(\lambda)<$ $\bar{c}(\lambda)=c_{I C h}$ for all $\lambda$.

\section{C.2 Case $\lambda>\widetilde{\lambda}$}

In this case we focus on $\left(\mathrm{ICh}_{B B}\right),(\mathrm{ICB})$ and (D) because the three conditions, with (ICB) binding, imply $\left(\mathrm{ICh}_{G B}\right)$. Recall that (ICB) holds for some $\mathbf{x}$ for large enough $\delta$ if 
and only if $c<c_{I C B}$, in which case $\hat{\mathbf{x}}=\left(1,1,1, \hat{x}_{G b}\right)$ maximizes $v_{F}(\mathbf{x})$ subject to (ICB), and binds $(\mathrm{ICB})$. It is straightforward to verify that $\left(\mathrm{ICh}_{B B}\right)$ holds at $\hat{\mathbf{x}}$ if and only if

$$
c \leq \widehat{c}(\lambda)=h-p_{B}-\lambda(1-h) \frac{p_{G}-p_{B}}{1-p_{B}}=\frac{(1-2 \lambda)^{2}(1-h) h^{2}}{(1-\lambda)(h+\lambda(1-2 h))(1-h-\lambda(1-2 h))} .
$$

Since $\widehat{c}(\lambda)<c_{I C B}$ and $\hat{\mathbf{x}}$ satisfies $(\mathrm{D})$, it follows that $\bar{v}_{F}=v_{F}(\hat{\mathbf{x}})$ if $c \leq \widehat{c}(\lambda)$, establishing Proposition 2 for $\lambda>\widetilde{\lambda}$ and $c \leq \widehat{c}(\lambda)$.

Next, for the remaining case that $c>\widehat{c}(\lambda)$ so that $\left(\mathrm{ICh}_{B B}\right)$ fails at $\hat{\mathbf{x}}$, consider a configuration $\mathbf{x}=\left(x_{B g}, x_{G g}, x_{B b}, x_{G b}\right)$ that supports $\bar{v}_{F}$, presuming it exists. First we show that

$$
x_{G g}=1 \text { or } x_{G b}=0
$$

must hold. To verify this, suppose to the contrary that $x_{G g}<1$ and $x_{G b}>0$. Then, one can increase $x_{G g}$ and decrease $x_{G b}$, while increasing $h \rho_{g}+(1-h) \rho_{b}$, i.e. $\pi_{\mathscr{g}} d x_{G g}+\left(1-\pi_{\mathfrak{g}}\right) d x_{G b}>$ 0 , and increasing $\pi_{\mathfrak{b}} \Delta_{g}+\left(1-\pi_{\mathfrak{b}}\right) \Delta_{b}$, i.e. $\pi_{\mathfrak{b}} d x_{G g}+\left(1-\pi_{\mathfrak{b}}\right) d x_{G b}<0$. This would increase $\bar{v}_{F}$ while relaxing (ICB), (D) and $\left(\mathrm{ICh}_{B B}\right)$, a contradiction. Hence, (24) must hold.

Also, we verify that

$$
\Delta_{b}=\Delta_{g} \quad \text { or } \quad x_{B g}=1 .
$$

Suppose otherwise, i.e., $\Delta_{b}>\Delta_{g}$ and $x_{B g}<1$. Then, since $\Delta_{b}>0$ by (ICB), one can reduce $x_{B b}$ and increase $x_{B g}$ while keeping $h \rho_{g}+(1-h) \rho_{b}$ constant, so that $h \lambda d x_{B g}+(1-$ $h)(1-\lambda) d x_{B b}=0 \Leftrightarrow \pi_{\mathfrak{b}} d x_{B g}+\left(1-\pi_{\mathfrak{b}}\right) d x_{B b}=0$ and $\ell d x_{B g}+(1-\ell) d x_{B b}<0$ because $\ell<\pi_{\mathfrak{b}}$ for $\lambda>\tilde{\lambda}$. This would keep $v_{F}(\mathbf{x})$ constant while relaxing $\left(\mathrm{ICh}_{B B}\right)$ and (ICB) because $d x_{B g}=d \Delta_{g}$ and $d x_{B b}=d \Delta_{b}$. As (D) remains slack, $v_{F}(\mathbf{x})$ can be increased above $\bar{v}_{F}$, a contradiction. Thus, (25) must hold.

Given (24) and (25), there are three possibilities in which $\mathbf{x}$ may support $\bar{v}_{F}:(i) x_{B g}=$ $x_{G g}=1,(i i) x_{G b}=0$ and $x_{B g}=1,(i i i) x_{G b}=0, \Delta_{b}=\Delta_{g}$ and $x_{B g}<1$. We examine these possibilities below.

We start with possibility $(i) x_{B g}=x_{G g}=1$, so that $\rho_{g}=1$. Solve binding $\left(\operatorname{ICh}_{B B}\right)$ and (ICB) simultaneously to get the solution:

$$
\begin{aligned}
\breve{x}_{B b} & =\frac{(1-\delta \ell)\left[\left(1-p_{B}\right)(h-\ell-c)-\left(p_{G}-p_{B}\right)(1-h) \lambda\right]-(1-\delta h)\left(1-p_{B}\right)\left(p_{B}-\ell\right)}{\delta(1-\ell)\left[\left(1-p_{B}\right)(h-\ell-c)-\left(p_{G}-p_{B}\right)(1-h) \lambda\right]-\delta(1-h)\left(1-p_{B}\right)\left(p_{B}-\ell\right)} \\
\breve{x}_{G b} & =\breve{x}_{B b}-\frac{\left(p_{G}-p_{B}\right)\left(1-\delta h-\delta(1-h) \breve{x}_{B b}\right)}{\delta\left[\left(1-p_{B}\right)(h-\ell-c)-\left(p_{G}-p_{B}\right)(1-h) \lambda\right]} .
\end{aligned}
$$

Note that $\breve{x}_{B b}$ can be rewritten as

$$
\breve{x}_{B b}=\frac{(1-\delta \ell)(\bar{c}(\lambda, \delta)-c)}{(1-\delta \ell)(\bar{c}(\lambda, \delta)-c)+(1-\delta)(c-\widehat{c}(\lambda))}
$$

where $\quad \bar{c}(\lambda, \delta)=h-\ell-\frac{(1-\delta h)\left(p_{B}-\ell\right)}{(1-\delta \ell)}-\lambda(1-h) \frac{p_{G}-p_{B}}{1-p_{B}}>\widehat{c}(\lambda)$. 
Hence, $\breve{x}_{B b} \in(0,1)$ if and only if $\widehat{c}(\lambda)<c<\bar{c}(\lambda, \delta)$. In this case, the initial formula of $\breve{x}_{B b}$ implies $\left(1-p_{B}\right)(h-\ell-c)-\left(p_{G}-p_{B}\right)(1-h) \lambda>0$ and thus, $\breve{x}_{G b}<\breve{x}_{B b}$ and both $\breve{x}_{B b}$ and $\breve{x}_{G b}$ converge to 1 from below as $\delta \rightarrow 1$. This implies that $\breve{\mathbf{x}}=\left(1,1, \breve{x}_{B b}, \breve{x}_{G b}\right)$ supports a FSGV that dominates any FSGV with $x_{G b}=0$ required by possibility $(i i)$ and $(i i i)$.

For $c \in(\widehat{c}(\lambda), \bar{c}(\lambda))$ and large enough $\delta$, therefore, $\bar{v}_{F}$ must be supported by a configuration $\mathbf{x}$ with $x_{G b}>0$ and moreover, $\left(\mathrm{ICh}_{B B}\right)$ binds at $\mathbf{x}$ by [C2] because $\hat{\mathbf{x}}$ fails $\left(\mathrm{ICh}_{B B}\right)$ in the current case. Since we may assume that (ICB) binds at a configuration that supports $\bar{v}_{F}$ by Lemma 1 , we deduce that $\breve{\mathbf{x}}=\left(1,1, \breve{x}_{B b}, \breve{x}_{G b}\right)$ supports $\bar{v}_{F}$ for large enough $\delta$, which is calculated (by Mathematica) as

$$
\bar{v}_{F}(\breve{\mathbf{x}})=-\frac{c(1-\ell)}{h-\ell}-\frac{\left[\begin{array}{c}
\ell(1-\lambda) \lambda^{2}+h^{2}(1-2 \lambda)\left(\lambda^{2}-\lambda+(1-\ell)(1-2 \lambda)\right) \\
-h\left(1-4 \lambda+5 \lambda^{2}-\lambda^{3}-\ell\left(1-3 \lambda+\lambda^{2}+2 \lambda^{3}\right)\right)
\end{array}\right]}{(h-\ell)(1-\lambda)\left[h(1-2 \lambda)^{2}(1-h)+\lambda(1-\lambda)\right]} h .
$$

This establishes Proposition 2 for $\lambda>\widetilde{\lambda}$ and $\widehat{c}(\lambda)<c<\bar{c}(\lambda)$.

It remains to consider $c>\bar{c}(\lambda)$ for $\lambda>\widetilde{\lambda}$. If $\bar{v}_{F}$ is supported by $\mathbf{x}$ that conforms to possibility $(i)$ but $x_{G b}>0$ (hence, neither $(i i)$ and $(i i i)$ ), then $\mathbf{x}$ must bind $\left(\mathrm{ICh}_{B B}\right)$ by [C2] because $\hat{\mathbf{x}}$ fails $\left(\mathrm{ICh}_{B B}\right)$, and $\mathbf{x}$ may also bind (ICB) by Lemma 1 but no such $\mathbf{x}$ exists as shown above.

Hence, it suffices to consider only $(i i)$ and $(i i i)$. We may assume that (ICB) binds by Lemma 1. If $\left(\mathrm{ICh}_{B B}\right)$ is slack, $x_{B b}=1$ must hold because otherwise raising $x_{B b}$ would increase $v_{F}(\mathbf{x})$ maintaining $(\mathrm{ICB})$ and $(\mathrm{D})$, a contradiction; but $x_{B b}=1$ is inconsistent with $(i i)$ because binding (ICB) would imply $v_{F}(\mathbf{x})-\underline{v} \rightarrow 0$ as $\delta \rightarrow 1$, contradicting $\left(\mathrm{ICh}_{B B}\right)$, nor is it with $($ iii $)$ because it would imply $\Delta_{b}=1>\Delta_{g}$.

Therefore, $\bar{v}_{F}$ should be supported by a configuration that binds both (ICB) and $\left(\mathrm{ICh}_{B B}\right)$, but we show this is impossible for large enough $\delta$ below, thus completing the proof of Proposition 2.

Possibility (ii): Suppose $x_{G b}=0$ and $x_{B g}=1$. Solving the simultaneous equation system consisting of the binding constraints $\left(\mathrm{ICh}_{B B}\right)$ and $(\mathrm{ICB})$, and evaluating the solution value of $x_{G g}$ at the limit $\delta=1$ gives

$$
\frac{(h-\ell-c)(1-\ell)-\left(p_{G}-p_{B}\right)(1-\ell)[h+\lambda-2 h \lambda]-\left(p_{B}-\ell\right)\left[1-h \lambda-p_{B}(h+\lambda-2 h \lambda)\right]}{(h-\ell-c) p_{B}(1-\ell)-\left(p_{G}-p_{B}\right)(1-\ell) h(1-\lambda)-\left(p_{B}-\ell\right)\left[h-h \lambda+p_{B}(1-2 h-\lambda+2 h \lambda)\right]}
$$

which obtains a value of 1 at $c=\bar{c}(\lambda)$. Moreover, its derivative w.r.t. $c$ is

$$
\frac{(1-\ell)\left[p_{B}(\lambda+h(1-2 \lambda))-h(1-\lambda)\right]\left[p_{B}^{2}-2 \ell p_{B}+p_{G}(\ell-1)+\ell\right]}{\left((h-\ell-c) p_{B}(1-\ell)-\left(p_{G}-p_{B}\right)(1-\ell) h(1-\lambda)-\left(p_{B}-\ell\right)\left[h(1-\lambda)+p_{B}(1-2 h-\lambda+2 h \lambda)\right]\right)^{2}}
$$


which is positive because i) $p_{B}(\lambda+h(1-2 \lambda))-h(1-\lambda)<0$ given $p_{B}<h$ if $\lambda>\widetilde{\lambda}$ and ii) $p_{B}^{2}-2 \ell p_{B}+p_{G}(\ell-1)+\ell<p_{B}^{2}-2 \ell p_{B}+h(\ell-1)+\ell<0$ for $\ell<p_{B}<h$. Thus, we have shown that the unique solution value of $x_{G g}$ to binding $\left(\mathrm{ICh}_{B B}\right)$ and (ICB) exceeds 1 for $c>\bar{c}(\lambda)$ and large enough $\delta$, hence no legitimate solution exists in the current case.

Possibility (iii): Suppose $x_{G b}=0, \Delta_{b}=\Delta_{g}$ and $x_{B g}<1$. Solving the simultaneous equation system consisting of the binding constraints $\left(\mathrm{ICh}_{B B}\right)$ and (ICB), and evaluating the solution value of $x_{B g}$ at the limit $\delta=1$ gives

$$
\left.x_{B g}\right|_{\delta=1}=\frac{c+p_{B}+\left(p_{G}-p_{B}\right)(\lambda-\ell+2 h(1-\lambda))-h}{c \ell+\ell\left(\ell-2 h-\left(p_{G}-p_{B}\right)(1-\lambda-h+2 h \lambda)\right)+h p_{G}} .
$$

The derivative of this w.r.t. $c$ is

$$
\frac{\left.\partial x_{B g}\right|_{\delta=1}}{\partial c}=\frac{(h-\ell)\left(p_{G}(1-\ell)+p_{B} \ell-\ell\right)}{\left[c \ell+\ell\left(\ell-2 h-\left(p_{G}-p_{B}\right)(1-\lambda-h+2 h \lambda)\right)+h p_{G}\right]^{2}}
$$

which is positive because $p_{G}(1-\ell)+p_{B} \ell-\ell$ exceeds its value at $p_{G}=h$ and $p_{B}=\ell$, namely $(h-\ell)(1-\ell)$, given $\lambda>\widetilde{\lambda}$.

Moreover, we calculate that (26) has a value of 1 at

$$
c=\frac{\ell(\ell-2 h)-\left(p_{G}-p_{B}\right)(2 h+(1-\ell)(1-2 h) \lambda-\ell h)+h\left(1+p_{G}\right)-p_{B}}{1-\ell} .
$$

Since subtracting this from $\bar{c}(\lambda)$ gives

$$
\frac{\left(h\left(1-p_{B}\right)-\lambda\left(p_{B}(1-2 h)+h\right)\right)\left(p_{G}-p_{B}\right)}{1-p_{B}}=\frac{(1-2 \lambda)(1-h) h\left(p_{G}-p_{B}\right)}{(1-h-\lambda(1-2 h))\left(1-p_{B}\right)}>0,
$$

(26) obtains a value of 1 at some $c<\bar{c}(\lambda)$ and thus exceeds 1 for $c \geq \bar{c}(\lambda)$. Hence, no legitimate solution exists for $c>\bar{c}(\lambda)$ and large enough $\delta$.

\section{Proof of Proposition 3}

Consider a non-trivial self-generated value, SGV for short, for which the seller may $\operatorname{mix} h$ and $\ell$ and/or report less than fully truthfully. We allow an arbitrary finite number of messages to encompass noisy communication of the signal as well as of the effort.

For any message $m$ used in the period strategy supporting the SGV, associated are continuation values denoted by $v_{m q}$ for each $q \in\{g, b\}$. The "(continuation) spread" of

message $m$ refers to $v_{m g}-v_{m b}$. Each $v_{m q}$ is between the SGV itself and $\underline{v}$, which we describe as being "feasible."

By an "agent e-s" we refer to a seller who exerted $e \in\{h, \ell\}$ and observed $s \in\{\mathfrak{g}, \mathfrak{b}\}$. Let $\pi_{s}^{e}=\pi_{s}$ if $e=h$ and $\pi_{s}^{e}=\pi_{s}^{\prime}$ if $e=\ell$. For each message $m$ used for a SGV, the seller's payoff from sending $m$ is linear in $\pi_{s}^{e}$ with a slope equal to the spread $\times \delta$ :

$$
U\left(m, \pi_{s}^{e}\right):=(1-\delta) p_{m}+\delta\left[\pi_{s}^{e}\left(v_{m g}-v_{m b}\right)+v_{m b}\right] .
$$


Imagine the upper envelope of all the graphs of $U(m, \pi)$ for all $m$ on $\pi \in\left[\pi_{\mathfrak{b}}^{\prime}, \pi_{\mathfrak{g}}\right]$. The optimal message(s) for each agent e-s are those whose graphs constitute the upper envelope at $\pi=\pi_{s}^{e}$. We may disregard any message whose graph is disjoint from the upper envelope. From the above, we have the following observations $[\mathrm{i}]-[\mathrm{v}]$ on the seller behavior supporting a SGV, that hold for all $\lambda \in(0,1 / 2)$.

[i] If agents e-s and e'-s' find it optimal to send $m$ and $m^{\prime}$, respectively, then the spread of $m$ is weakly larger than that of $m^{\prime}$ if $\pi_{s}^{e} \geq \pi_{s^{\prime}}^{e^{\prime}}$ from the discussion above, or equivalently, because

$$
\begin{aligned}
(1-\delta) p_{m}+\delta\left[\pi_{s}^{e} v_{m g}+\left(1-\pi_{s}^{e}\right) v_{m b}\right] & \geq(1-\delta) p_{m^{\prime}}+\delta\left[\pi_{s}^{e} v_{m^{\prime} g}+\left(1-\pi_{s}^{e}\right) v_{m^{\prime} b}\right] \\
(1-\delta) p_{m}+\delta\left[\pi_{s^{\prime}}^{e^{\prime}} v_{m g}+\left(1-\pi_{s^{\prime}}^{e^{\prime}}\right) v_{m b}\right] & \leq(1-\delta) p_{m^{\prime}}+\delta\left[\pi_{s^{\prime}}^{e^{\prime}} v_{m^{\prime} g}+\left(1-\pi_{s^{\prime}}^{e^{\prime}}\right) v_{m^{\prime} b}\right] \\
\Longrightarrow \pi_{s}^{e}\left[\left(v_{m g}-v_{m b}\right)-\left(v_{m^{\prime} g}-v_{m^{\prime} b}\right)\right] & \geq \pi_{s^{\prime}}^{e^{\prime}}\left[\left(v_{m g}-v_{m b}\right)-\left(v_{m^{\prime} g}-v_{m^{\prime} b}\right)\right] .
\end{aligned}
$$

[ii] Each used message is optimal for an "adjacent" set of agents, i.e., all agents with $\pi_{s}^{e}$ in a certain interval. Multiple messages optimal for multiple agents must have the same graph, hence same spread, $v_{m g}-v_{m b}$, and same intercept, $(1-\delta) p_{m}+\delta v_{m b}$.

[iii] Any two messages $m$ and $m^{\prime}$ optimal for all agents in a given set of agents can be replaced, without affecting optimality conditions, by a new message obtained by the convex combination of $m$ and $m^{\prime}$ with weights equal to their respective probabilities relative to total probability. Hence, one may assume at most one message that is optimal for and only for all agents in any given "adjacent" subset of agents. ${ }^{19}$ Moreover, if such a message exists for an adjacent set of agents, then it is the unique message commonly optimal for any non-singleton subset of those agents.

[iv] If $h$ is exerted with positive probability, the spread of any message optimal for the agent $h-\mathbb{g}$ is positive: otherwise, the spread of every used message would be nonpositive and thus, the upper envelope of the graphs of all messages is non-positively sloped. This would mean that the optimal expected payoff is no lower for an $\ell$-seller than for an $h$-seller so that exerting $h$ is suboptimal considering the cost $c>0$, contrary to $h$ being exerted.

[v] Consider two used messages $m$ and $m^{\prime}$ with associated prices $p_{m}$ and $p_{m^{\prime}}$, respectively, such that $p_{m}<p_{m^{\prime}}$ and the spread of $m^{\prime}$ is larger than that of $m$ which is positive. Consider a third message $m^{\prime \prime}$ with $p_{m}<p_{m^{\prime \prime}}<p_{m^{\prime}}$ followed by continuation values $v_{m^{\prime \prime} q}=v_{m q}-(1-\delta)\left(p_{m^{\prime \prime}}-p_{m}\right) / \delta$ for $q \in\{g, b\}$. Then, the expected payoff from

\footnotetext{
${ }^{19}$ Note that this doesn't prevent that several messages be optimal for any given agent.
} 
sending $m$ and $m^{\prime \prime}$ are identical for all agents and $v_{m^{\prime \prime} q}$ is feasible. It is feasible because the agent who uses $m$ must weakly prefer sending $m^{\prime \prime}$ to $m^{\prime}$, which implies, together with both the price and the spread being larger for $m^{\prime}$ than $m^{\prime \prime}$, that $v_{m^{\prime \prime} q} \geq v_{m^{\prime} b}$.

With these observations at hand, we now show that there is no SGV larger than $\max \left\{v^{*}, \bar{v}_{F}\right\}$ in Step 1 and Step 2 below. Then, we show that all values in $\left[\underline{v}, \max \left\{v^{*}, \bar{v}_{F}\right\}\right]$ can be achieved as equilibrium values in Step 3.

STEP 1: Mixing $h$ and $\ell$ does not increase the seller's value.

We will show that for any SGV for which $h$ and $\ell$ are mixed, there is a weakly higher SGV supported by a period strategy where effort is not mixed, if $\delta<1$ is large enough.

Lemma 3 Consider a $S G V v^{\prime}>\underline{v}$ for which $h$ and $\ell$ are mixed.

(a) If a message $G^{\prime}$ is used by agent $h-\mathrm{g}$ with $p_{G^{\prime}} \leq \pi_{\mathfrak{g}}$ and $B^{\prime} \neq G^{\prime}$ is used by agent $h-b$ with $p_{B^{\prime}} \leq \pi_{\mathfrak{b}}$, then there is a weakly higher $S G V$ for which effort is not mixed.

(b) If a message $G^{\prime}$ is used by agent $\ell-g$ and $B^{\prime} \neq G^{\prime}$ is used by agent $\ell-b$, then either $p_{G^{\prime}}>\pi_{\mathfrak{g}}^{\prime}$ or $p_{B^{\prime}}>\pi_{\mathfrak{b}}^{\prime}$.

Proof. Part (a). Consider a SGV $v^{\prime}>\underline{v}$ as above. We build a weakly higher SGV supported by a period strategy in which $h$ is exerted for sure, and alternative messages $G^{\prime \prime}$ and $B^{\prime \prime}$ with prices $p_{G^{\prime \prime}} \leq \pi_{\mathrm{g}}$ and $p_{B^{\prime \prime}}=\pi_{\mathfrak{b}}$, together with suitably modified continuation values. Recall that payoff from sending message $m$ depends only on $(1-\delta) p_{m}+\delta v_{m b}$ and the spread $v_{m g}-v_{m b}$ (observation [i]).

If $p_{G^{\prime}}=\pi_{\mathfrak{g}}$ and $p_{B^{\prime}}=\pi_{\mathfrak{b}}$, then $v^{\prime}$ can be supported by the seller exerting $h$ for sure and sending $G^{\prime}\left(B^{\prime}\right)$ after good (bad) signal, i.e., without mixing $h$ and $\ell$. Hence, suppose $p_{G^{\prime}}<\pi_{\mathfrak{g}}$ or $p_{B^{\prime}}<\pi_{\mathfrak{b}}$, and consider hypothetical messages denoted by $G_{d}^{\prime}$ and $B_{d}^{\prime}$ with associated prices higher than $p_{G^{\prime}}$ and $p_{B^{\prime}}$, respectively, by the same amount $d>0$, keeping the continuation values unchanged. Increase $d$ until either $p_{B_{d}^{\prime}}$ hits $\pi_{\text {b }}$ or $p_{G_{d}^{\prime}}$ hits $\pi_{\mathrm{g}}$.

Case 1. $\pi_{\mathfrak{g}}-p_{G^{\prime}} \geq \pi_{\mathfrak{b}}-p_{B^{\prime}}$ : In this case, $p_{B_{d}^{\prime}}$ hits $\pi_{\mathfrak{b}}$, say at $d=d_{1}$, before $p_{G_{d}^{\prime}}$ hits $\pi_{\mathfrak{g}}$. At this point, given the two messages $B_{d_{1}}^{\prime}$ and $G_{d_{1}}^{\prime}$, the seller finds it optimal to exert $h$ and report $B_{d_{1}}^{\prime}$ after $s=\mathbb{b}$ and $G_{d_{1}}^{\prime}$ after $s=\mathfrak{g}$ (because the payoff from exerting $h$ increased by $\pi_{\mathfrak{b}}-p_{B^{\prime}}$ and that from $\ell$ increased by no more). This generates a value strictly larger than $v^{\prime}$ and all continuation values are clearly "feasible" since they did not change. Note also that $p_{G_{d_{1}}^{\prime}}>p_{G^{\prime}}>\pi_{\mathbb{b}}$ because $G^{\prime}$ is sent by no agent $e-s$ with $\pi_{s}^{e}<\pi_{\text {b }}$ to self-generate $v^{\prime}$ initially (observation [iii]).

Now, increase $p_{G_{d}^{\prime}}$ until either it reaches $\pi_{\mathfrak{g}}$ or agent $h$-b becomes indifferent between $B_{d_{1}}^{\prime}$ and $G_{d}^{\prime}$. At that point, exerting $h$ is optimal conditional on $M=\left\{G_{d}^{\prime}, B_{d_{1}}^{\prime}\right\}$ (because an $\ell$-seller benefits less from increased $p_{G_{d}^{\prime}}$ given that it announces $B_{d_{1}}^{\prime}$ if $s=\mathbb{b}$ and thus 
announces $G_{d}^{\prime}$ less often than an $h$-seller) and $h$-seller's value is $v^{\prime \prime}>v^{\prime}$. In the case that $p_{G_{d}^{\prime}}$ reaches $\pi_{\mathrm{g}}$ first, $v^{\prime \prime}$ is the value of an equilibrium in which the seller exerts $h$ for sure and sends $B_{d_{1}}^{\prime}$ after $s=\mathbb{b}$ and $G_{d}^{\prime}$ after $s=\mathfrak{g}$. In the alternative case that $p_{G_{d}^{\prime}}<\pi_{\mathrm{g}}$ reaches a point at which agent $h$-b becomes indifferent between $B_{d_{1}}^{\prime}$ and $G_{d}^{\prime}$, $v^{\prime \prime}$ is an equilibrium value where agent $h$-b mixes between $B_{d_{1}}^{\prime}$ and $G_{d}^{\prime}$ appropriately so that $p_{G_{d}^{\prime}}$ is the price for the message $G_{d}^{\prime}$ obtained by Bayes rule. In either case, by Property 1 (p.12), $v^{\prime \prime}$ is SGV supported by a period strategy in which $h$ is exerted for sure as desired.

Case 2. $\pi_{\mathfrak{g}}-p_{G^{\prime}}<\pi_{\mathfrak{b}}-p_{B^{\prime}}$ : Next, consider the case that $p_{G_{d}^{\prime}}$ hits $\pi_{\mathfrak{g}}$ first at $d=d_{1}$. If the spread of $B^{\prime}$ is positive, then use observation [v] to replace message $B_{d_{1}}^{\prime}$ by an equivalent message $B^{\prime \prime}$ with price $p_{B^{\prime \prime}}=\pi_{\mathfrak{b}}\left(\right.$ use $m=G_{d_{1}}^{\prime}, m^{\prime}=B_{d_{1}}^{\prime}$ and $p_{m^{\prime \prime}}=\pi_{\mathfrak{b}}$ in [v]). Then the SGV $v^{\prime}$ can be supported with effort $h$ only and messages $G^{\prime \prime}=G_{d_{1}}^{\prime}$ and $B^{\prime \prime}$.

If the spread of $B^{\prime}$ is negative, consider a modified message $B_{d}^{\prime}$ by increasing $p_{B_{d}^{\prime}}$ toward $\pi_{\mathrm{b}}$ while decreasing $\delta v_{B_{d}^{\prime} g} /(1-\delta)$ and $\delta v_{B_{d}^{\prime} b} /(1-\delta)$ by the same amount to keep $B_{d}^{\prime}$ to be "equivalent" with $B_{d_{1}}^{\prime}$ for every agent. If $p_{B_{d}^{\prime}}$ reaches $\pi_{\mathfrak{b}}$ before $v_{B_{d}^{\prime} g}$ hits $\underline{v}$, a higher equilibrium value is supported by a seller exerting $h$ for sure and reporting $B_{d}^{\prime}$ when $s=\mathbb{b}$ and and $G_{d_{1}}^{\prime}$ when $s=\mathfrak{g}$ (because the payoff should have increased weakly more for $h$-seller than for $\ell$-seller), which is also a SGV by Property 1.

In the alternative case that $v_{B_{d}^{\prime} g}$ hits $\underline{v}$ before $p_{B_{d}^{\prime}}$ reaches $\pi_{\mathfrak{b}}$, say at $d=d_{2}$. Continue to increase $p_{B_{d}^{\prime}}$ to $\pi_{\mathfrak{b}}$ while decreasing $v_{B_{d}^{\prime} b}$ to keep the payoff of agent $h$-b from sending $B_{d}^{\prime}$, i.e. $(1-\delta) p_{B_{d}^{\prime}}+\delta \pi_{\mathfrak{b}}\left(\underline{v}-v_{B_{d}^{\prime} b}\right)+\delta v_{B_{d}^{\prime} b}$, constant. Note that $p_{B_{d}^{\prime}}$ reaches $\pi_{\mathfrak{b}}$, say at $d=d_{3}>d_{2}$, before $v_{B_{d}^{\prime} b}$ hits $\underline{v}$, because otherwise the payoff of agent $h$-b from sending $B_{d}^{\prime}$ would be less than $(1-\delta) \pi_{\mathfrak{b}}+\delta \underline{v}$ which is strictly less than that from sending $G^{\prime}$. During the process from $d_{2}$ to $d_{3}$, the payoff from sending $B_{d}^{\prime}$ decreases for $\pi<\pi_{\mathfrak{b}}$ and increases for $\pi>\pi_{\mathfrak{b}}$.

At $d=d_{3}$, the payoff of exerting $h$ (and announcing $B_{d_{3}}^{\prime}$ after $s=\mathbb{b}$ and $G_{d_{1}}^{\prime}$ after $s=\mathfrak{g}$ ) remains unchanged since $d=d_{1}$. On the other hand, the maximum payoff from exerting $\ell$ decreased if $\lambda \geq \tilde{\lambda}$, because then given $\pi_{\mathfrak{b}}^{\ell}<\pi_{\mathfrak{g}}^{\ell} \leq \pi_{\mathfrak{b}}$ sending $B_{d}^{\prime}$ is optimal for both $\ell$ - $g$ and $\ell$ - $b$ and the payoff from it remained the same for $d \in\left(d_{1}, d_{2}\right)$ but decreased after $d=d_{2}$ as noted above. Even when $\lambda<\widetilde{\lambda}$ so that $\pi_{\mathfrak{b}}^{\ell}<\pi_{\mathfrak{b}}<\pi_{\mathrm{g}}^{\ell}$, if $\ell$-seller's payoff increased after $d=d_{2}$, then $B_{d}^{\prime}$ should be optimal at $d=d_{3}$ for agent $\ell$-g as well as $\ell$-b, implying that the payoff from sending $B_{d}^{\prime}$ regardless of the signal, $(1-\delta) p_{B_{d}^{\prime}}+\delta \ell\left(\underline{v}-v_{B_{d}^{\prime} b}\right)+\delta v_{B_{d}^{\prime} b}$, increased for $d \in\left(d_{2}, d_{3}\right)$. For this, since $(1-\delta) p_{B_{d}^{\prime}}+\delta\left(1-\pi_{\mathfrak{b}}\right) v_{B_{d}^{\prime} b}$ remains constant for $d \in\left(d_{2}, d_{3}\right)$, we would need $\delta\left(\pi_{\mathfrak{b}}-\ell\right) v_{B_{d}^{\prime} b}$ increase, implying that $\pi_{\mathfrak{b}}<\ell$. But, then $\ell$-seller's payoff from sending $B_{d}^{\prime}$ at $d=d_{3}$, denoted by $v$, would have to satisfy

$$
v \leq(1-\delta) \ell+\delta \ell(\underline{v}-v)+\delta v \leq(1-\delta) \ell+\delta v \Longrightarrow v \leq \ell=\underline{v}
$$


contradicting $v \geq v^{\prime}>\underline{v}$.

Hence, at $d=d_{3}$ with $M=\left\{G_{d_{1}}^{\prime}, B_{d_{3}}^{\prime}\right\}$ and continuation values specified above, exerting $h$ must be optimal, followed by $B_{d_{3}}^{\prime}$ after $s=\mathfrak{b}$ and $G_{d_{1}}^{\prime}$ after $s=\mathfrak{g}$. As this constitutes an equilibrium with a value $v^{\prime \prime}>v^{\prime}$ and continuation values lower than $v^{\prime \prime}$, by Property 1 $v^{\prime \prime}$ is a FSGV as desired.

Part $(b)$. If $p_{G^{\prime}} \leq \pi_{\mathfrak{g}}^{\prime}$ and $p_{B^{\prime}} \leq \pi_{\mathfrak{b}}^{\prime}$, the value $v^{\prime}$ from optimally exerting $\ell$ and sending messages $G^{\prime}$ and $B^{\prime}$ after respective signals is at most

$$
(1-\delta)\left[(\ell(1-\lambda)+(1-\ell) \lambda) \pi_{\mathfrak{g}}^{\prime}+(\ell \lambda+(1-\ell)(1-\lambda)) \pi_{\mathfrak{b}}^{\prime}\right]+\delta v^{\prime} \leq(1-\delta) \ell+\delta v^{\prime},
$$

leading to $v^{\prime} \leq \ell=\underline{v}$, a contradiction.

Consider an arbitrary SGV $v^{\prime}>\underline{v}$ for which $h$ and $\ell$ are mixed. Suppose there is a message, say $H$, used by both agents $h$-g and $h$-b in the period strategy supporting $v^{\prime}$ such that $p_{H} \leq h$. When $H$ is modified by increasing the price to $h$ with the same continuation values, it remains optimal for the seller to exert $h$ and send the message $H$ regardless of signal, which constitutes an equilibrium with a higher value. By Property 1, therefore, a higher SGV exists for which effort is not mixed.

Hence, suppose that $p_{H}>h$ for any message $H$ used by both agents $h$ - $g$ and $h$-b (in the period strategy supporting $v^{\prime}$ ). Clearly, $p_{G^{\prime}} \leq \pi_{\mathrm{g}}$ for any message $G^{\prime}$ used by agent $h$-g. If there is a message used by agent $h$ - $\mathfrak{b}$, say $B^{\prime}$, such that $p_{B^{\prime}} \leq \pi_{\mathfrak{b}}$, there is a SGV $v^{\prime \prime} \geq v^{\prime}$ for which effort is not mixed by Lemma 3 (a).

Hence, suppose that $p_{B^{\prime}}>\pi_{\mathfrak{b}}$ for any message $B^{\prime}$ used by $h$-b, so that it must be shared by an agent with a higher posterior on the item's quality. If all such messages are shared by agent $h$-g but not by $\ell$-g, which must the case if $\lambda \geq \widetilde{\lambda}$ so that $\pi_{\mathfrak{g}}^{\prime} \leq \pi_{\mathfrak{b}}<\pi_{\mathfrak{g}}$, then not all of them may carry prices exceeding $h$ since the mean of those prices cannot exceed $h$, contrary to the supposition above.

Thus, assume $\lambda<\tilde{\lambda}$ (so that $\pi_{\mathfrak{b}}<\pi_{\mathfrak{g}}^{\prime}<\pi_{\mathfrak{g}}$ ) and thus, all messages used by $h$-b are shared by $\ell$-g. Suppose any of them, say $H$, is also shared by $h$-g, whence agent $\ell$-g uses $H$ for sure by [iii]. ${ }^{20}$ If agent $h$-b also uses $H$ for sure, we would have $p_{H} \leq \max \left\{\pi_{\mathfrak{g}}^{\prime}, h\right\}$, necessitating $h<\pi_{\mathfrak{g}}^{\prime}$ for $p_{H}>h$. Then, agent $\ell$-b should use $H$ for sure as well due to Lemma $3(\mathrm{~b})$, contradicting $p_{H}>h$. If agent $h$-lb uses a message different from $H$, on the other hand, the associated price is no higher than $\pi_{\mathfrak{b}}$ as it is not shared by $\ell-\mathfrak{g}$ or $h-\mathfrak{g}$, and a higher SGV exists for which effort is not mixed by Lemma 3 (a).

In the remaining case that $h$-b shares every message with $\ell$ - $g$ but not with $h$ - $\mathfrak{g}$, the associated price is at most $\pi_{\mathfrak{g}}^{\prime}$ and thus, by Lemma 3 (b) agent $\ell$-b must share all messages

\footnotetext{
${ }^{20}$ As no other message than $H$ can be shared with types $h$-g and $h$-bo, any other message used by $\ell-g$ would be preferred to $H$ by type $h-\mathrm{g}$ or type $h-b$.
} 
with agents $h$-b and $\ell$-g. For any such message (unique by [iii]), say $L$, we have $p_{L} \leq$ $\max \left\{\ell, \pi_{\mathfrak{b}}\right\}$. Recall that we assumed $\pi_{\mathfrak{b}}<p_{L}$ for a message played by type $h$-b which implies that $p_{L} \leq \ell$. Then, since exerting $\ell$ is optimal in the period strategy, we would have $v^{\prime} \leq(1-\delta) p_{L}+\delta v^{\prime} \leq(1-\delta) \ell+\delta v^{\prime}$ so that $v^{\prime} \leq \ell$, contradicting $v^{\prime}>\underline{v}$.

STEP 2: Not fully truthful announcements do not increase the seller's value

We now consider SGV's for which effort is not mixed in the period strategy. If the effort exerted is $\ell$, the associated SGV is clearly $\ell=\underline{v}$. We show below that any SGV $v_{h}(>\underline{v})$ for which the seller exerts $h$ for sure but does not report the signal fully truthfully, is no higher than $\max \left\{v^{*}, \bar{v}_{F}\right\}$ if $c<h-\ell$ and $\delta$ is large enough.

By observation [iii], we may consider only up to three used messages with at most one of them being sent by both agent $h$-g and agent $h$-bb. Hence, there are three possibilities to consider as below.

Possibility 1: Suppose that an $h$-seller always announces a message $G$ after $s=\mathfrak{g}$, but after $s=\mathbb{b}$ she announces $G$ and $B$ with probability $\theta \in(0,1)$ and $1-\theta$, respectively. We call the associated SGV a semi-faithfully SGV. Then, the price remains at $p_{B}$ after $m=B$ but changes to

$$
p_{G}^{\theta}:=p_{G}-\frac{\kappa(\theta)}{h(1-\lambda)+(1-h) \lambda}>h>p_{B}>\ell
$$

after $m=G$ where

$$
\kappa(\theta):=\frac{h(1-h)(1-2 \lambda) \theta}{h(1-\lambda)+(1-h) \lambda+(h \lambda+(1-h)(1-\lambda)) \theta}<h(1-h)
$$

and the value (computed from truthful reporting out of indifference) is

$$
v_{h}(\mathbf{x})=\frac{(1-\delta)(h-\ell-c-\kappa(\theta))}{1-\delta\left(h \rho_{g}+(1-h) \rho_{b}\right)}+\underline{v}
$$

We assume $h-\ell-c-\kappa(\theta)>0$ because $v_{h}(\mathbf{x})>\underline{v}$.

The maximum semi-faithfully SGV, which we denote by $\bar{v}_{h}$ if exists, is the solution value to the program

$$
\bar{v}_{h}=\max _{\mathbf{x} \in[0,1]^{4}} v_{h}(\mathbf{x})
$$

subject to $\left(\mathrm{ICh}_{G B}\right),\left(\mathrm{ICh}_{B B}\right),(\mathrm{ICB})$ and $(\mathrm{D})$ with $p_{G}$ replaced by $p_{G}^{\theta}$ and $v_{F}(\mathbf{x})$ by $v_{h}(\mathbf{x})$, and the additional restriction that $(\mathrm{ICB})$ binds at $\mathbf{x}$.

In the proof of Lemma 1 , to prove that $\bar{v}_{F}$ is supported by a configuration that binds (ICB), the actual value of $p_{G}$ was not used but only the fact that $p_{G} \in(h, 1)$. Therefore, Lemma 1 extends to establish that the maximum semi-faithfully SGV, $\bar{v}_{h}$, is also the 
solution value to the program (27) without requiring that (ICB) bind at $\mathbf{x}$, which is how we treat (27) from now on. Then, various results on $\bar{v}_{F}$ extend to $\bar{v}_{h}$ as described below.

We start with the observation that $\bar{v}_{h}$ is dominated by $\bar{v}_{F}$ for large enough $\delta$ if the latter also exists:

Lemma 4 If $c<\bar{c}(\lambda)$ then $\bar{v}_{h} \leq \bar{v}_{F}$ for large enough $\delta$ if $\bar{v}_{h}$ exists.

Proof. Consider a maximum semi-faithfully SGV $\bar{v}_{h}$ supported by a configuration $\mathbf{x}$ such that $x_{G b} \rightarrow 1$ as $\delta \rightarrow 1$ (because otherwise $\bar{v}_{h}<\bar{v}_{F}$ for large enough $\delta$ by Proposition 2). By observations [i]-[v] above, the payoff from sending $m=B$ is $U(B, \pi)=(1-\delta) p_{B}+$ $\delta\left[\pi\left(v_{B g}-v_{B b}\right)+v_{B b}\right]$ and that from sending $m=G$ is $U(G, \pi)=(1-\delta) p_{G}^{\theta}+\delta\left[\pi\left(v_{G g}-\right.\right.$ $\left.\left.v_{G b}\right)+v_{G b}\right]$ such that $U\left(B, \pi_{\mathfrak{b}}\right)=U\left(G, \pi_{\mathfrak{b}}\right)$ and $v_{G g}-v_{G b}>\max \left\{0, v_{B g}-v_{B b}\right\}$. Since $x_{G b} \rightarrow 1 \Leftrightarrow v_{G b} \rightarrow \bar{v}_{h}$ as $\delta \rightarrow 1$, one can find $v_{G b}^{\prime} \in\left(\underline{v}, v_{G b}\right)$ such that

$$
(1-\delta)\left(\pi_{\mathfrak{g}}-p_{G}^{\theta}\right)=\delta\left(1-\pi_{\mathfrak{g}}\right)\left(v_{G b}-v_{G b}^{\prime}\right)
$$

so that $(1-\delta) \pi_{\mathfrak{g}}+\delta\left[\pi\left(v_{G g}-v_{G b}^{\prime}\right)+v_{G b}^{\prime}\right]$ is equal to $U(G, \pi)$ at $\pi=\pi_{\mathfrak{g}}$ but lower than $U(G, \pi)$ at $\pi<\pi_{\mathfrak{g}}$. Hence, when $p_{G}^{\theta}$ is replaced by $p_{G}=\pi_{\mathfrak{g}}$ and $v_{G b}$ by $v_{G b}^{\prime}$, the seller would find the faithful strategy optimal and thus, $\bar{v}_{h}$ can be generated as a FSGV.

By the same reasoning as in [C1] of the proof of Proposition 2, (ICB) must hold at $(1,1,1,0)$ for any $\mathbf{x}$ to bind (ICB), which is the case for large $\delta$ only if

$$
c<c_{I C B}^{\theta}:=h-\ell-\kappa(\theta)-\lambda(1-h) \frac{p_{G}^{\theta}-p_{B}}{1-p_{B}}<c_{I C B}
$$

where the latter inequality follows from

$$
-\kappa(\theta)+\frac{\lambda(1-h) \kappa(\theta)}{\left(1-p_{B}\right)(h(1-\lambda)+(1-h) \lambda)}<0
$$

because $\frac{\lambda(1-h)}{\left(1-p_{B}\right)(h(1-\lambda)+(1-h) \lambda)}<\left.\frac{\lambda(1-h)}{\left(1-p_{B}\right)(h(1-\lambda)+(1-h) \lambda)}\right|_{p_{B}=h}<1$. In addition, for $\lambda \leq \tilde{\lambda}$, analogously to the first paragraph of Case C.1 in the proof of Proposition 2, $\left(\mathrm{ICh}_{G B}\right)$ may hold for some $\mathbf{x}$ only if

$$
c<c^{*}+(1-2 \lambda) \frac{(h-\ell)(1-h)}{1-\ell}\left(p_{G}^{\theta}-p_{B}\right)-\kappa(\theta) \frac{h-\ell}{1-\ell}<c_{I C h} .
$$

Hence, for $\lambda \leq \widetilde{\lambda}$, if a semi-faithfully SGV exists then $c<\bar{c}(\lambda)$ and thus, $\bar{v}_{h} \leq \bar{v}_{F}$ by Lemma 4.

Next, consider the case that $\lambda>\widetilde{\lambda}$. Assume $c<c_{I C B}^{\theta}$ so that (ICB) is satisfied at some $\mathbf{x}$, thus at $\mathbf{x}=(1,1,1,0)$. Again, the arguments for the Case C.2 in the proof of 
Proposition 2 extend to the current case with $p_{B}$ replaced by $p_{B}^{\theta}$ and $v_{F}(\mathbf{x})$ by $v_{h}(\mathbf{x})$. In particular, (ICB) holding at $\mathbf{x}=(1,1,1,0)$ implies that $v_{h}(\mathbf{x})$ is maximized subject to $(\mathrm{ICB})$ and $(\mathrm{D})$ at $\hat{\mathbf{x}}^{\theta}=\left(1,1,1, \hat{x}_{G b}^{\theta}\right)$ that binds $(\mathrm{ICB})$. Since $\left(\mathrm{ICh}_{B B}\right)$ is satisfied at $\hat{\mathbf{x}}^{\theta}$ if and only if

$$
c \leq \widehat{c}^{\theta}(\lambda)=h-p_{B}-\kappa(\theta)-\lambda(1-h) \frac{p_{G}^{\theta}-p_{B}}{1-p_{B}}<\widehat{c}(\lambda)
$$

where the latter inequality is due to $(28)$, it follows that $\bar{v}_{h}=v_{h}\left(\hat{\mathbf{x}}^{\theta}\right)$ if $c \leq \hat{c}^{\theta}(\lambda)$, in which case $\bar{v}_{h} \leq \bar{v}_{F}$ by Lemma 4 because $\widehat{c}(\lambda)<\bar{c}(\lambda)$ for $\lambda>\widetilde{\lambda}$.

In addition, for $c>\widehat{c}^{\theta}(\lambda)$, the arguments in Case C.2 establish that (24) and (25) must hold at the solution $\mathbf{x}$ to (27), leaving three cases to consider: $(i) x_{B g}=x_{G g}=1$, (ii) $x_{G b}=0$ and $x_{B g}=1,($ iii $) x_{G b}=0, \Delta_{b}=\Delta_{g}$ and $x_{B g}<1$. The analyses for these cases also extend straightforwardly with suitable modifications as summarized below. Used in this process is the claim [C2] which is straightforwardly verified to hold for $\bar{v}_{h}$ as well.

For the case $(i) x_{B g}=x_{G g}=1$, the solution values $\breve{x}_{B b}$ and $\breve{x}_{G b}$ that bind both (ICB) and $\left(\mathrm{ICh}_{B B}\right)$ are of the same formulae as before with $c$ replaced by $c+\kappa(\theta)$ and $p_{G}$ by $p_{G}^{\theta}$, thus a legitimate solution exists only if

$$
c<\bar{c}^{\theta}(\lambda)=h-\ell-\kappa(\theta)-\frac{(1-\delta h)\left(p_{B}-\ell\right)}{(1-\delta \ell)}-\lambda(1-h) \frac{p_{G}^{\theta}-p_{B}}{1-p_{B}}<\bar{c}(\lambda)
$$

where the inequality follows from (28). In this case, $\left(\mathrm{ICh}_{B B}\right)$ must bind at a configuration supporting $\bar{v}_{h}$ by [C2], where (ICB) also binds by Lemma 1 . Thus, $\left(1,1, \breve{x}_{B b}, \breve{x}_{G b}\right)$ supports $\bar{v}_{h}$ if $c \in\left(\widehat{c}^{\theta}(\lambda), \bar{c}^{\theta}(\lambda)\right)$, whence $\bar{v}_{h} \leq \bar{v}_{F}$ by Lemma 4 .

For the remaining case that $\lambda>\widetilde{\lambda}$ and $c \geq \bar{c}^{\theta}(\lambda)$, we may focus on possibilities $(i i)$ and (iii) and $\left(\mathrm{ICh}_{B B}\right)$ should bind at the configuration that supports $\bar{v}_{h}$ for the same reasoning as in Case C.2 of proof of Proposition 2, where we may assume (ICB) also binds by Lemma 1. But, such a configuration does not exist for large enough $\delta$ as asserted below.

For the possibility $(i i) x_{G b}=0$ and $x_{B g}=1$, the solution value $\left.x_{G g}\right|_{\delta=1}$ obtains a value of 1 at $c=\left.\bar{c}^{\theta}(\lambda)\right|_{\delta=1}<\bar{c}(\lambda)$ and increases in $c$ because its derivative w.r.t. $c$ is of the same formula as in C.2 with $c$ replaced by $c+\kappa(\theta)$ and $p_{G}$ by $p_{G}^{\theta}$. Hence, no legitimate solution exists. For $(i i i) x_{G b}=0, \Delta_{b}=\Delta_{g}$ and $x_{B g}<1$, again the suitably modified solution value of $x_{B g}$ increases in $c$ and exceeds 1 at $c=\bar{c}^{\theta}(\lambda)$ by the same reasoning, precluding any legitimate solution.

Possibility 2: Suppose that a SGV $v_{h}$ is supported by an $h$-seller who always announces a message $B$ after $s=\mathbb{b}$, but announces $G$ and $B$ with probability $\theta \in(0,1)$ and $1-\theta$, respectively, after $s=\mathfrak{g}$. Then, the price remains at $p_{G}$ after $m=G$ while it is $p_{B}^{\theta} \in\left(p_{B}, h\right)$ after $m=B$. 
As exerting $h$ and reporting $B$ is optimal for the seller, her value is

$$
\begin{aligned}
& v_{h}=(1-\delta)\left(p_{B}^{\theta}-c\right)+\delta\left(h x_{B g}+(1-h) x_{B b}\right)\left(v_{h}-\underline{v}\right)+\delta \underline{v} \\
& \Longrightarrow \quad v_{h}=\frac{(1-\delta)\left(p_{B}^{\theta}-\ell-c\right)}{1-\delta\left(h x_{B g}+(1-h) x_{B b}\right)}+\underline{v}
\end{aligned}
$$

so that, in particular, we need $p_{B}^{\theta}>\ell$ for $v_{h}>\underline{v}$. As an $\ell$-seller could always report $B$, optimality of exerting $h$ requires

$$
\delta(h-\ell)\left(x_{B g}-x_{B b}\right)\left(v_{h}-\underline{v}\right) \geq(1-\delta) c
$$

subject to which $v_{h}$ is maximized at $x_{B g}=1$ and $x_{B b}$ that binds the inequality.

Notice from (ICh), however, that this is the condition for $\left(e_{1}, \rho_{g}, \rho_{b}\right)$ to constitute a self-generated value without communication where $e_{1}$ satisfies $p_{B}^{\theta}=e_{1}(h-\ell)+\ell$ and $\left(\rho_{g}, \rho_{b}\right)=\left(x_{B g}, x_{B b}\right)$. As shown in Section 2, therefore, $v_{h} \leq v^{*}$ if $c<c^{*}$ and $v_{h}=\underline{v}$ if $c \in\left[c^{*}, h-\ell\right)$.

Possibility 3: The remaining possibility is that an $h$-seller sends $G$ and a third message $m$ after $s=\mathfrak{g}$, and $B$ and $m$ after $s=\mathbb{b}$ for a SGV. Then, $p_{B}<p_{m}<p_{G}$ and we must have

$$
\begin{aligned}
(1-\delta) p_{B}+\delta\left[\pi_{\mathfrak{b}} v_{B g}+\left(1-\pi_{\mathfrak{b}}\right) v_{B b}\right] & =(1-\delta) p_{m}+\delta\left[\pi_{\mathfrak{b}} v_{m g}+\left(1-\pi_{\mathfrak{b}}\right) v_{m b}\right] \\
& \geq(1-\delta) p_{G}+\delta\left[\pi_{\mathfrak{b}} v_{G g}+\left(1-\pi_{\mathfrak{b}}\right) v_{G b}\right] \\
\text { and } \quad(1-\delta) p_{G}+\delta\left[\pi_{\mathfrak{g}} v_{G g}+\left(1-\pi_{\mathfrak{g}}\right) v_{G b}\right] & =(1-\delta) p_{m}+\delta\left[\pi_{\mathfrak{g}} v_{m g}+\left(1-\pi_{\mathfrak{g}}\right) v_{m b}\right] \\
& \leq(1-\delta) p_{B}+\delta\left[\pi_{\mathfrak{g}} v_{B g}+\left(1-\pi_{\mathfrak{g}}\right) v_{B b}\right] .
\end{aligned}
$$

Therefore, if both $G$ and $B$ are sent with positive probability, the same value is generated by $h$-seller who sends $G$ and $B$ with certainty after $s=\mathfrak{g}$ and $s=\mathbb{b}$, respectively, i.e., through a faithful strategy, with the same continuation values. Note that the seller cannot benefit by exerting $\ell$ instead of $h$ with message $m$ removed, because the expected payoffs from sending $G$ and $B$ remain the same for $\ell$-seller. If both $G$ and $B$ are unused, on the other hand, it amounts to babbling and SGV's of this kind have been covered in Section 2. The case that only $G$ or only $B$ is unused amounts to Possibility 1 and Possibility 2 above, respectively.

STEP 3: every value in $\left[v, \max \left\{v^{*}, \bar{v}_{F}\right\}\right]$ is an equilibrium value

In STEPs 1-2 above, we have shown that $\max \left\{v^{*}, \bar{v}_{F}\right\}$ is the tight upper bound of all SGV for large enough $\delta<1$. When $v^{*}=\max \left\{v^{*}, \bar{v}_{F}\right\}$, the claim has been shown already in Proposition 1. 
Hence, consider the alternative case, i.e., $\bar{v}_{F}>v^{*}$, implying that $c<\bar{c}(\lambda)$. From the proof of Proposition 2, there exists $v_{0}<\bar{v}_{F}$ such that a continuum $\left[v_{0}, \bar{v}_{F}\right]$ of FSGV's exists for large enough $\delta$.

Consider the following strategy: exert $\ell$ in the first $t$ periods, followed by a continuation value $v \in\left[v_{0}, \bar{v}_{F}\right]$, generating a value of $\ell\left(1-\delta^{t}\right)+\delta^{t} v=\ell\left(1-\delta^{t}\right)+\delta^{t}(v-\underline{v})+\delta^{t} \underline{v}=$ $\underline{v}+\delta^{t}(v-\underline{v})$. Given the price of $\ell$ in the first $t$ periods followed by such a continuation value $v$, it is optimal to exert $\ell$ in the first $t$ periods. The set of values that can be generated with $t$ lags as such is $\left[\underline{v}+\delta^{t}\left(v_{0}-\underline{v}\right), \underline{v}+\delta^{t}\left(\bar{v}_{F}-\underline{v}\right)\right]$. Note that $\underline{v}+\delta^{t+1}\left(\bar{v}_{F}-\underline{v}\right)-\underline{v}-\delta^{t}\left(v_{0}-\underline{v}\right)=$ $\delta^{t}\left(\delta \bar{v}_{F}-v_{0}+\underline{v}-\delta \underline{v}\right)>0$ where the inequality holds if $\delta$ is large enough. Therefore, every value in $\left(\underline{v}, v_{0}\right)$ is a FSGV with $t$ lags for some $t$ if $\delta$ is large enough. Consequently, every value in $\left(\underline{v}, \bar{v}_{F}\right]$ is either a FSGV or a FSGV with $t$ lags for some $t$ if $\delta$ is large enough, all of which constitute equilibrium values (with public randomization).

This proves Step 3, thus completing the proof of Proposition 3.

\section{E. Proof of Proposition 4}

From Proposition $2\left(i i^{\prime}\right)$, the most efficient equilibrium is achieved at $\overline{\mathbf{x}}$ that binds both constraints (18) and (19). Hence, communication is beneficial if $\bar{\rho}_{b}=(1-\lambda) \bar{x}_{B b}+\lambda \bar{x}_{G b}>\rho_{b}^{*}$ at this solution. To facilitate comparison, we rearrange $\rho_{b}^{*}$ in (6) as

$$
1-\rho_{b}^{*}=\frac{c}{(h-\ell)^{2}-c(1-\ell)}\left(\frac{1-\delta}{\delta}\right)=\frac{c}{c^{*}-c} \times \frac{1-\delta}{\delta(1-\ell)} \quad \text { for } c<c^{*} .
$$

Similarly, we solve for $\bar{x}_{B b}$ and $\bar{x}_{G b}$ from (18) and (19) and express $\bar{\rho}_{b}$ as

$$
1-\bar{\rho}_{b}=\frac{\Lambda(\lambda)+c}{\Lambda(\lambda) \frac{h-1}{1-\ell}+c^{*}-c} \times \frac{1-\delta}{\delta(1-\ell)} \text { for } \lambda>\widetilde{\lambda} \text { and } \widehat{c}(\lambda)<c<\bar{c}(\lambda)
$$

where

$$
\Lambda(\lambda):=\lambda\left(\frac{p_{G}-p_{B}}{1-p_{B}}\right)(1-\ell)-h+p_{B}=\frac{c^{*}-\bar{c}(\lambda)}{1-h} .
$$

Note that $1-\bar{\rho}_{b}$ is an increasing function of $\Lambda(\lambda)$ and is equal to $1-\rho_{b}^{*}$ when $\Lambda(\lambda)=0$. Thus, whether $\bar{\rho}_{b}$ exceeds $\rho_{b}^{*}$ or not is independent of $c$ in the current case. Specifically, for $\lambda>\widetilde{\lambda}$ and $c \in\left(0, c^{*}\right) \cap(\widehat{c}(\lambda), \bar{c}(\lambda))$, we have

$$
\bar{\rho}_{b}>\rho_{b}^{*} \Longleftrightarrow \Lambda(\lambda)<0 \Longleftrightarrow c^{*}<\bar{c}(\lambda) \Longleftrightarrow \underline{c}(\lambda)<\widehat{c}(\lambda)
$$

where the last equivalence follows from $\bar{c}(\lambda)-c^{*}=(1-h)(\widehat{c}(\lambda)-\underline{c}(\lambda))$. It is verified straightforwardly that $\bar{c}(\lambda)$ decreases in $\lambda>\widetilde{\lambda}$ and hits $c^{*}$ at

$$
\lambda=\bar{\lambda}:=\frac{(1-h)(3 h-\ell)}{2(2 h-1)(h-\ell)}\left(\sqrt{1+\frac{4(2 h-1)(h-\ell) h}{(3 h-\ell)^{2}(1-h)}}-1\right)>\tilde{\lambda}^{21}
$$

\footnotetext{
${ }^{21}$ If $h=\frac{1}{2}$, then $\bar{\lambda}=\frac{1}{3-2 \ell}>\widetilde{\lambda}$.
} 
Consequently, together with an earlier assertion that $\widehat{c}(\lambda)<\bar{c}(\lambda)$ for $\lambda>\widetilde{\lambda}$, we deduce that

$$
\begin{cases}\lambda \in(\widetilde{\lambda}, \bar{\lambda}) & \Longrightarrow\left(\widehat{c}(\lambda), c^{*}\right) \subset(\underline{c}(\lambda), \bar{c}(\lambda)) \text { and } \bar{\rho}_{b}>\rho_{b}^{*} \text { on } c \in\left(\widehat{c}(\lambda), c^{*}\right) \\ \lambda \geq \bar{\lambda} & \Longrightarrow(\underline{c}(\lambda), \bar{c}(\lambda)) \subset\left(\widehat{c}(\lambda), c^{*}\right) \quad \text { and } \quad \bar{\rho}_{b} \leq \rho_{b}^{*} \text { on } c \in(\widehat{c}(\lambda), \bar{c}(\lambda))\end{cases}
$$

We now combine (31) with the condition (16) for beneficial communication when (17) holds. First, (16) implies communication is beneficial when $\underline{c}(\lambda)<c<\widehat{c}(\lambda)$ if $\lambda \in(\widetilde{\lambda}, \bar{\lambda})$, but is vacuous if $\lambda \geq \bar{\lambda}$ because $\widehat{c}(\lambda)<\underline{c}(\lambda)$ by (30). For $\lambda<\widetilde{\lambda}$, (16) implies beneficial communication for $c \in(\underline{c}(\lambda), \bar{c}(\lambda)) \cap\left(0, c^{*}\right)$. Finally, when $c \geq c^{*}$, communication is beneficial whenever a FSGV exists, i.e., $c \leq \bar{c}(\lambda)$.

We deduce that $\bar{v}_{F}>v^{*}$ for large enough $\delta$ if and only if $\lambda<\bar{\lambda}$ and $\min \left\{\underline{c}(\lambda), c^{*}\right\}<$ $c<\bar{c}(\lambda)$. To conclude the proof it suffices to show that

$$
\left\{c \mid \min \left\{\underline{c}(\lambda), c^{*}\right\}<c<\bar{c}(\lambda)\right\}=\{c \mid \underline{c}(\lambda)<c<\bar{c}(\lambda)\}
$$

For $\lambda \leq \tilde{\lambda}$, this follows from $c^{*}<\underline{c}(\lambda) \Leftrightarrow \bar{c}(\lambda)<c^{*}$ because

$$
\bar{c}(\lambda)=h-\ell-\lambda(1-h) \frac{p_{G}-p_{B}}{1-p_{B}}<c^{*}=\frac{(h-\ell)^{2}}{1-\ell}
$$

is equivalent to

$$
\frac{(h-\ell)(1-h)}{1-\ell}<\lambda(1-h) \frac{p_{G}-p_{B}}{1-p_{B}} \Leftrightarrow \frac{(h-\ell)^{2}}{1-\ell}<\lambda(1-h) \frac{p_{G}-p_{B}}{1-p_{B}}=\underline{c}(\lambda) .
$$

For $\lambda>\widetilde{\lambda}$, we verify (32) by showing $\underline{c}(\lambda)<c^{*}$ below. First, observe that

$$
\underline{c}^{\prime}(\lambda)=\frac{h(h-\ell)\left(h(1-2 \lambda)^{2}-\lambda^{2}\right)}{(1-\lambda)^{2}(\lambda+h-2 \lambda h)^{2}}
$$

is positive for $\lambda<\breve{\lambda}$ and negative for $\lambda>\breve{\lambda}$, hence $\underline{c}(\lambda)$ is single-peaked at $\breve{\lambda}=\frac{\sqrt{h}}{1+2 \sqrt{h}} \in$ $(0,1 / 3)$ with a maximum $\underline{c}(\breve{\lambda})=\frac{h(h-\ell)}{(1+\sqrt{h})^{2}}$.

From $\underline{c}(\widetilde{\lambda})=h(h-\ell) \frac{\sqrt{h(1-h)(1-\ell) \ell}-(1-h) \ell}{(1-h) \sqrt{h(1-h)(1-\ell) \ell}+h^{2}(1-\ell)}$, we have

$$
\frac{c^{*}-\underline{c}(\widetilde{\lambda})}{h-\ell}=\frac{\left(h^{2}+\ell-2 h \ell\right)[\sqrt{h(1-h)(1-\ell) \ell}-(1-\ell) h]}{(\ell-1)\left[(1-h) \sqrt{h(1-h)(1-\ell) \ell}+h^{2}(1-\ell)\right]}>0
$$

where the inequality follows from $\sqrt{h(1-h)(1-\ell) \ell}<(1-\ell) h$. Hence, $\underline{c}(\lambda)<c^{*}$ is verified for $\lambda>\widetilde{\lambda}$ if $\breve{\lambda} \leq \widetilde{\lambda}$. 
Finally, $\breve{\lambda}>\widetilde{\lambda}$ if and only if $\ell<\frac{h^{2}}{1+2(1-h) \sqrt{h}}$. Moreover, $\frac{c^{*}-\underline{c}(\breve{\lambda})}{h-\ell}=\frac{h-\ell}{1-\ell}-\frac{h}{(1+\sqrt{h})^{2}}$ is decreasing in $\ell$ and assumes a positive value of $2 \sqrt{h^{3}} /(1+\sqrt{h})^{2}$ at $\ell=\frac{h^{2}}{1+2(1-h) \sqrt{h}}$. Therefore, $\underline{c}(\lambda)<c^{*}$ obtains for $\lambda>\widetilde{\lambda}$ when $\breve{\lambda}>\widetilde{\lambda}$ as well.

\section{F. Proof of Lemma 2}

Observe that

$$
\bar{\lambda}=\frac{\sqrt{1+2 Y}-1}{(3-\beta) Y} \text { where } Y=\frac{2(2 h-1)(1-\beta)}{(1-h)(3-\beta)^{2}} \text { and } \beta=\ell / h .
$$

Note that $\bar{\lambda} \rightarrow 1 / 2$ as $\beta \rightarrow 1$ because $\left.Y\right|_{\beta=1}=0$ and $\frac{\sqrt{1+2 Y}-1}{Y}=\frac{2}{\sqrt{1+2 Y}+1} \rightarrow 1$ as $Y \rightarrow 0$. In addition,

$$
\frac{\partial \bar{\lambda}}{\partial \beta}=\frac{1}{(1-\beta)(3-\beta)^{2} \sqrt{1+\frac{2 Y}{(3-\beta)}}}\left[2 \frac{1+2 Y-\sqrt{1+2 Y}}{Y}-1-\beta\right] .
$$

Note that the fraction in the bracket is positive and increasing in $Y>-0.5$, and that $Y>\left.Y\right|_{h=0}=\frac{-2(1-\beta)}{(3-\beta)^{2}}>-0.5$ since $Y$ increases in $h$. Thus, the expression in the bracket is minimal at $h=0$ for any given $\beta$, which is calculated as

$$
2 \frac{1+\left.2 Y\right|_{h=0}-\sqrt{1+\left.2 Y\right|_{h=0}}}{\left.Y\right|_{h=0}}-1-\beta=(3-\beta) \frac{\sqrt{5-2 \beta+\beta^{2}}-2}{1-\beta}>0
$$

where the inequality follows because $5-2 \beta+\beta^{2}>4$. This proves that $\partial \bar{\lambda} / \partial \beta>0$.

This proves that $\bar{\lambda}$ increases in $\ell$. Also, as $\bar{\lambda}$ decreases in $h$ for given $\beta$ and $\bar{\lambda}$ increases in $\beta$ which decreases in $h, \bar{\lambda}$ decreases in $h$. 


\section{References}

Abreu, D., D. Pearce, and E. Stacchetti (1986), "Optimal Cartel Monitoring with Imperfect Information," Journal of Economic Theory, 39, 251-269.

Abreu, D., D. Pearce, and E. Stacchetti (1990), "Toward a Theory of Discounted Repeated Games with Imperfect Monitoring," Econometrica, 58, 1041-1063.

Agnihotri, R., Rapp, A. and Trainor, K. (2009). "Understanding the role of information communication in the buyer-seller exchange process: antecedents and outcomes," Journal of Business \&f Industrial Marketing, 24, 474-486.

Athey, S. and Bagwell, K. (2001), "Optimal collusion with private information," RAND Journal of Economics, 32, 428-465.

Athey, S., Bagwell, K., and Sanchirico, C. (2004) "Collusion and price rigidity," Review of Economic Studies, 71, 317-349.

Awaya, Y., and Krishna, V. (2016). "On Communication and Collusion," American Economic Review, 106, 285-315.

Bar-Isaac, H., (2003), "Reputation and Survival: learning in a dynamic signalling model," Review of Economic Studies, 70, 231-251.

Baker, G., Gibbons, R. and Murphy, K. J. (2002), "Relational Contracts and the Theory of the Firm," Quarterly Journal of Economics, 117, 39-84.

Ben-Porath, E. and Kahneman, M. (1996), "Communication in repeated games with private monitoring." Journal of Economic Theory, 70, 281-297.

Best, J. and Quigley, D. (2017), "Persuasion for the Long Run," mimeo, University of Oxford.

Board, S., and Meyer-ter-Vehn, M. (2013). "Reputation for quality." Econometrica, 81, 2381-2462.

Cabral, L., and Hortacsu, A. (2010). "The dynamics of seller reputation: Evidence from eBay." The Journal of Industrial Economics, 58, 54-78.

Compte, O. (1998), "Communication in Repeated Games with Imperfect Private Monitoring," Econometrica, 66, 597-626.

Dellarocas, C. (2003). "The Digitization of Word of Mouth: Promise and Challenges of Online Feedback Mechanisms," Management Science, 49, 1407-1424.

Fudenberg, D. and Levine, D. (1992), "Maintaining a Reputation When Strategies Are Imperfectly Observed," Review of Economic Studies, 59, 561-579.

Fudenberg, D. and Levine, D. (1994), "Efficiency and Observability with Long-run and Short-run Players," Journal of Economic Theory, 62, 103-135.

Fudenberg, D., Levine, D. and Maskin, E., (1994), "The Folk Theorem with Imperfect Public Information," Econometrica, 62, 997-1039. 
Green, E. and Porter, R. (1984), "Noncooperative Collusion under Imperfect Price Information," Econometrica, 52, 87-100.

Inderst, R. and Ottaviani, M. (2009). "Misselling through agents" American Economic Review, 99, 883-908.

Jullien, B. and Park, I.-U. (2014), "New, Like New, or Very Good? Reputation and Credibility," Review of Economic Studies, 81, 1543-1574.

Kamenica, E. and Gentzkow, M. (2011), "Bayesian Persuasion," American Economic Review, 101, 2590-2615.

Kandori, M. (2003), "Randomization, Communication, and Efficiency in Repeated Games with Imperfect Public Monitoring," Econometrica, 71, 345-353.

Klein, Tobias J., Christian Lambertz, and Stahl, K. (2016), "Market transparency, adverse selection, and moral hazard," Journal of Political Economy, 124, 1677-1713.

Klein, B. and Leffler, K. (1981). "The role of market forces in assuring contractual performances," Journal of Political Economy, 89, 615-641.

Kreps, D. and Wilson, R. (1982), "Reputation and Imperfect Information," Journal of Economic Theory, 27, 245-252.

Levin, J. (2003), "Relational incentive contracts," American Economic Review, 93, 835857.

MacLeod, W. B. (2007) "Reputations, relationships, and contract enforcement," Journal of Economic Literature, 45, 595-628.

MacLeod, W. B. and Malcomson, J.M. (1989), "Implicit Contracts, Incentive Compatibility, and Involuntary Unemployment," Econometrica, 57, 447-80.

Mailath, G. and Samuelson, L., (2001), "Who Wants a Good Reputation," Review of Economic Studies, 68, 415-441.

Milgrom, P. and Roberts, D.J. (1982), "Predation, Reputation and Entry Deterrence," Journal of Economic Theory, 27, 280-312.

Morris, S. (2001). "Political correctness," Journal of political Economy, 109, 231-265.

Palmatier, R.W., Dant, R.P., Grewal, D. and Evans, K.R. (2006), "Factors influencing the effectiveness of relationship marketing: a meta-analysis," Journal of Marketing, 70, 136-53.

Porter, R. (1983) "Optimal Cartel Trigger Price Strategies," Journal of Economic Theory, 29, 313-338.

Rhodes, A., and Wilson, C. (2016), "False Advertising," mimeo, TSE.

Shapiro, C. (1983). "Premiums for high quality products as returns to reputations," Quarterly Journal of Economics, 98, 659-679.

Sobel, J. (1985). "A theory of credibility," Review of Economic Studies, 52, 557-573.

Tadelis, S. (2016), "Reputation and Feedback Systems in Online Platform Markets," $A n$ nual Review of Economics, 8, 321-340. 